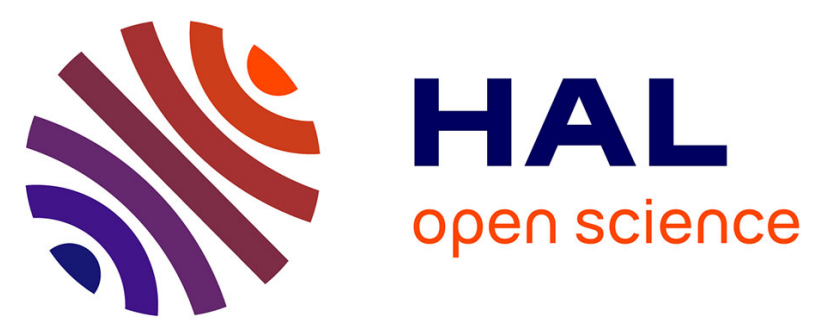

\title{
Carbon-13 Heteronuclear Longitudinal Spin Relaxation for Geometrical (and Stereochemical) Determinations in Small or Medium Size Molecules
}

Daniel Canet, Sabine Bouguet-Bonnet, Sébastien Leclerc, Mehdi Yemloul

\section{- To cite this version:}

Daniel Canet, Sabine Bouguet-Bonnet, Sébastien Leclerc, Mehdi Yemloul. Carbon-13 Heteronuclear Longitudinal Spin Relaxation for Geometrical (and Stereochemical) Determinations in Small or Medium Size Molecules. Annual Reports on NMR Spectroscopy, 2011, 74, pp.89-123. 10.1016/B9780-08-097072-1.00002-9 . hal-01482005

\section{HAL Id: hal-01482005 \\ https://hal.univ-lorraine.fr/hal-01482005}

Submitted on 3 Mar 2017

HAL is a multi-disciplinary open access archive for the deposit and dissemination of scientific research documents, whether they are published or not. The documents may come from teaching and research institutions in France or abroad, or from public or private research centers.
L'archive ouverte pluridisciplinaire HAL, est destinée au dépôt et à la diffusion de documents scientifiques de niveau recherche, publiés ou non, émanant des établissements d'enseignement et de recherche français ou étrangers, des laboratoires publics ou privés.

\section{(ㅇ)(1) $\$$}

Distributed under a Creative Commons Attribution - NonCommercial - NoDerivatives 44.0 


\title{
Carbon-13 Heteronuclear Longitudinal Spin Relaxation for Geometrical (and Stereochemical) Determinations in Small or Medium Size Molecules
}

\author{
Daniel Canet ${ }^{1}$, Sabine Bouguet-Bonnet ${ }^{1}$, Sébastien Leclerc ${ }^{2}$, Mehdi Yemloul ${ }^{1}$
}

\begin{tabular}{|c|c|}
\hline Contents & 1. Introduction. \\
\hline & 2. Overview of nuclear longitudinal spin relaxation.. \\
\hline & 2.1 Dipolar interaction........... \\
\hline & 2.2 Chemical shift anisotropy....... \\
\hline & 3. Cross-relaxation and cross-correlation. Solomon equations.................. 9 \\
\hline & 3.1 Simple Solomon equations............................... \\
\hline & 3.2 Longitudinal spin order. Extended Solomon equations.... \\
\hline & 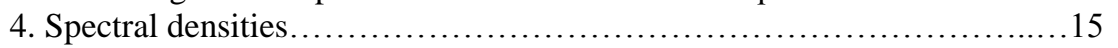 \\
\hline & 4.1 Isotropic tumbling of a rigid molecule............................... \\
\hline & 4.2 Anisotropic tumbling of a rigid molecule.... \\
\hline & 4.3 Local motions. The model free approach.................. \\
\hline & 5. Experimental procedures.................................. \\
\hline & 5.1 Measurement of ${ }^{13} \mathrm{C}$ longitudinal relaxation times..................22 \\
\hline & 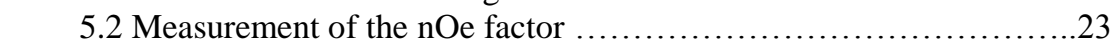 \\
\hline & 5.3 The HOESY experiment.......... \\
\hline & 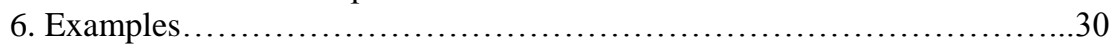 \\
\hline & 6.1 Stereochemical and conformational studies......................... 30 \\
\hline & 6.2 Geometrical determinations.................. \\
\hline & 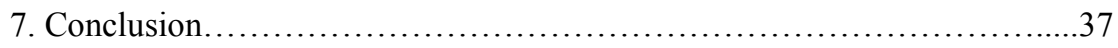 \\
\hline & Acknowledgements........... \\
\hline & References............ \\
\hline
\end{tabular}

\begin{abstract}
Owing to an extremely abundant literature making use of spin relaxation for structural studies, this review is limited to carbon-13 spectroscopy, to small or medium size molecules, to stereochemical and preferably geometrical determinations. The parameter of choice is evidently the Nuclear Overhauser effect (nOe) because it depends exclusively on the dipolar interaction mechanism, thus on $1 / r^{6}$, where $r$ is the distance between the two interacting spins. However, it depends also on the dynamical features of the system under investigation which must be characterized prior to any attempt for obtaining geometrical or stereochemical information. Therefore, this review is devoted not only to ${ }^{1} \mathrm{H}-{ }^{13} \mathrm{C}$ nOe but, more generally, to ${ }^{13} \mathrm{C}$ longitudinal relaxation. After comprehensive theoretical developments, experimental methods presently available will be presented. The latter include the usual gated decoupling experiment and pulse experiments of the HOESY (Heteronuclear Overhauser Effect SpectroscopY) family. These pulse experiments, which imply carbon-13 observation, can be one-dimensional, selective one-dimensional or two-dimensional. The emphasis will be put on the interpretation which is different according to the occurrence or not of extreme narrowing conditions. Along with a literature survey, some selected examples will be presented in detail in order to illustrate the potentiality of the method.
\end{abstract}

1 Méthodologie RMN (CRM²; UMR 7036, UHP-CNRS), Faculté des Sciences et Techniques, B.P. 239, 54506 Vandœuvre-lèsNancy (cedex), France

2 LEMTA (UMR 7563, Nancy-Université-CNRS), BP 160, 54504 Vandoeuvre-lès-Nancy (cedex), France

Keywords : carbon-13 spin relaxation, $T_{1}$ measurements, nuclear Overhauser effect, rotation-diffusion tensor, HOESY experiments 


\section{Introduction}

Spin relaxation in NMR is known to provide information about the dynamics of molecular entities and possibly about molecular geometry or electron distribution. Generally, dynamical information is obtained if the tensor of the relevant relaxation mechanism is known from independent determinations. Conversely, if parameters describing the dynamics of the considered molecule have been deduced beforehand, geometrical parameters may be derived. Only in particular situations, one can hope to access both types of parameters (dynamical and geometrical). For instance, this can occur when relaxation parameters become frequency dependent (i.e. dependent on the static magnetic field value at which measurements are performed). This method, sometimes dubbed "relaxometry", may yield, independently of the relaxation mechanism details, a so-called "spectral density mapping" which contains the major dynamical features of the considered molecule. In turn, when inserted in the theoretical expression of a given relaxation rate (the inverse of the corresponding relaxation time), it can provide some geometrical parameters.

The dipolar interaction (or, in other terms, the interaction between the magnetic moments associated with two nuclear spins) is the relaxation mechanism of choice when one attempts to access interatomic distances (and sometimes bond angles). As it will be explained later, this mechanism brings a contribution to relaxation rates proportional to $1 / r^{6}$, where $r$ is the distance between the two interacting spins. The problem is that the two usual relaxation times depend on other relaxation mechanisms and that it is not always easy to separate the dipolar contribution. Let us recall that the two usual relaxation times include $T_{1}$, the longitudinal or spin-lattice relaxation time, which refers to the recovery of the longitudinal nuclear magnetization component (the component along the static magnetic field direction) and $T_{2}$, the transverse or spin-spin relaxation time, which 
refers to the decay of nuclear magnetization components perpendicular to $B_{0}$, the static magnetic field. $T_{1}$ and $T_{2}$ are involved in Bloch equations ${ }^{1}$ which predict a monoexponential evolution of the nuclear magnetization components. It turns out that, when two spins $1 / 2, A$ and $B$, are coupled by dipolar interactions, their longitudinal relaxation is no longer mono-exponential. The longitudinal components of their magnetizations (or rather their polarizations) are coupled by a cross-relaxation rate denoted by $\sigma_{A B}$ which depends solely on their mutual dipolar interaction. Hence, the interest brought to this parameter from which arises the so-called nuclear Overhauser effect $^{2}$ (nOe) from Overhauser who discovered that polarization of an electron spin could be partly transferred to a nuclear spin through a cross-relaxation rate. Note that we are interested here in mutual transfers from one nuclear spin to another nuclear spin. Of course, there is an enormous literature about proton-proton nOe, especially through the NOESY ${ }^{3}$ (Nuclear Overhauser Effect SpectroscopY) two-dimensional experiment. The latter is routinely used for determining distance correlations in all types of molecules, noticeably in macromolecules of biological interest (proteins, nucleic acids...), these correlations being invaluable in view of determining, for instance, the tertiary structure of proteins. In the present review, we shall limit ourselves to ${ }^{13} \mathrm{C}-{ }^{1} \mathrm{H}$ nOe and more especially to the exploitation of cross-relaxation rates in view of geometrical (or stereochemical) determinations. This means that we also disregard the classical use of the heteronuclear nOe factor which enables us to evaluate the amount of non dipolar contributions to the longitudinal relaxation rates $^{4}$. For achieving such an objective, a firm theoretical background is required. It will be provided in section 2 by a brief overview of longitudinal nuclear spin relaxation and, in section 3, by the presentation of Solomon equations which constitute the basis of nOe studies. We shall also see in this section that, besides crossrelaxation rates, cross-correlation rates, which couple polarizations and longitudinal order, 
can be accounted for in extended Solomon equations. In section 4, we shall concentrate on spectral densities which represent the dynamical part of cross-relaxation or crosscorrelation rates and which must be considered carefully if geometrical or stereochemical information has to be derived from cross-relaxation or cross-correlation rates. Sections 3 and 4 represent actually a sort of manual for interpreting heteronuclear (intramolecular) proton-carbon-13 relaxation data. Experimental procedures for measuring ${ }^{13} \mathrm{C}-{ }^{1} \mathrm{H}$ crossrelaxation or cross-correlation rates will be presented in section 5, while some selected examples will be detailed in section 6 along with a survey of literature on the subject of the present review.

\section{Overview of longitudinal nuclear spin relaxation ${ }^{5,6}$}

At thermal equilibrium, the nuclear magnetization $M_{0}$ lies along the $\mathrm{B}_{0}$ direction, generally denoted as $z$. When nuclear magnetization has been taken out from its equilibrium state (with the help of a radio-frequency field), its longitudinal component $M_{z}$ tends to recover toward $M_{0}$. As for any physical system, this is a relaxation phenomenon which should originate from a perturbation comparable to the radiofrequency field which has been responsible for the non equilibrium situation. The latter is time dependent and perfectly coherent. Moreover, it acts at a well defined frequency. Conversely, a given nuclear spin is subjected within the sample to randomly fluctuating magnetic fields arising from other spins or from whatever interaction that the considered spin could experience. These randomly fluctuating fields $b(t)$ could play the same role as radio-frequency field, but in a reverse way, in order to restore the longitudinal magnetization toward its equilibrium value. These random fields are effectively time dependent with a zero mean value: $\overline{b(t)}=0$ (actually, the bar denotes an ensemble 
average). They should also present some degree of coherence which can be evaluated by the so-called correlation function $\overline{b(t) b(0)}$. This can be seen from the following $a$ contrario argument: if the random field at time $t$ is independent of the random field at time zero, one has $\overline{b(t) b(0)}=\overline{b(t)} \overline{b(0)}=0$. Thus the correlation function is the key to the efficiency of a given relaxation mechanism. It remains to determine at which frequencies this correlation function is active. This feature is deduced from the Fourier transform of the correlation function. This latter quantity is called spectral density and can be expressed as

$$
J(\omega) \propto \int_{-\infty}^{+\infty} \overline{b(t) b(0)} e^{-i \omega t} d t
$$

As we shall see, all relaxation rates are expressed as linear combinations of spectral densities. We shall retain the two relaxation mechanisms which are involved in the present study: the dipolar interaction and the so-called chemical shift anisotropy (csa) which can be important for carbon-13 relaxation. We shall disregard all other mechanisms because it is very likely that they will not affect carbon-13 relaxation. Let us denote by $R_{1}$ the inverse of $T_{1} . R_{1}$ governs the recovery of the longitudinal component of polarization, $I_{z}$, and, of course, the usual nuclear magnetization which is simply the nuclear polarization times the gyromagnetic constant $\lambda$. The relevant evolution equation is one of the famous Bloch equations ${ }^{1}$, valid, in principle, for a single spin but which, in many cases, can be used as a first approximation.

$$
\frac{d I_{z}}{d t}=-R_{1}\left(I_{z}-I_{e q}\right)
$$

\subsection{Dipolar interaction}

The classical interaction energy of two magnetic dipoles oriented along the direction of the static magnetic field (figure 1) is given by 


$$
E_{d}=\frac{\mu_{A} \mu_{B}}{r_{A B}^{3}}\left(3 \cos ^{2} \theta-1\right)
$$

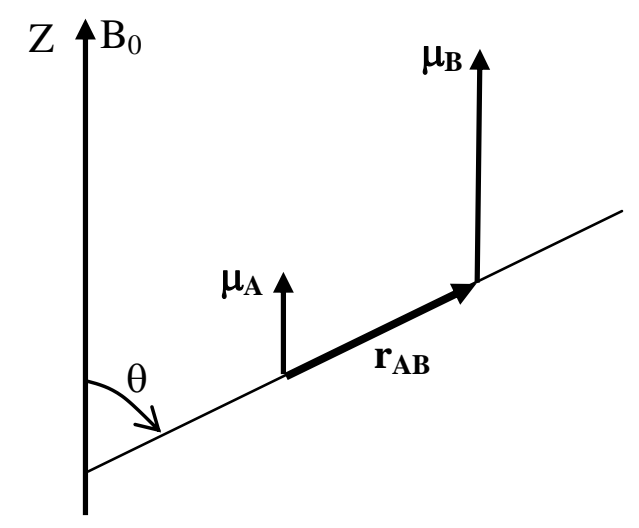

Figure 1. Magnetic dipoles associated with nuclear spins in the presence of $\mathrm{B}_{0}$.

As a consequence, the local field acting on $\mu_{A}$ is of the form

$$
b(t) \propto \frac{\mu_{B}}{r_{A B}^{3}}\left[3 \cos ^{2} \theta(t)-1\right]
$$

since the time dependence arises from the angle $\theta$ and is related to the molecular reorientation (molecular tumbling and possibly internal motions). As a matter of fact, the problem must be treated by quantum mechanics so as to introduce the proper spin operators and, doing so, we obtain the following spectral density actually involved in relaxation rates

$$
\begin{aligned}
& J_{d}(\omega)=\frac{K_{d}}{r_{A B}^{6}} \tilde{J}(\omega) \\
& \tilde{J}(\omega)=\frac{5}{4} \int_{-\infty}^{+\infty}\left[\overline{\left.\cos ^{2} \theta(t)-1\right]\left[3 \cos ^{2} \theta(0)-1\right]} e^{-i \omega t} d t\right.
\end{aligned}
$$

In (5), $\widetilde{J}(\omega)$ is independent of the relaxation mechanism and the constant $K_{d}$ is given by

$$
K_{d}=(1 / 20)\left(\mu_{0} / 4 \pi\right)^{2}\left(\gamma_{A} \gamma_{B} \hbar\right)^{2}
$$

$\mu_{0}$ is the vacuum permeability, $\gamma_{A}$ and $\gamma_{B}$ the gyromagnetic constants, $\hbar$ the Planck constant divided by $2 \pi$. If $A$ stands for a carbon- 13 and $B$ for a proton, $K_{d}$ is equal to 1.8 
$10^{9}$ provided that, in (4) and (5), $r$ is expressed in $\AA$.

In (5), we can first notice i) the factor $1 / r^{6}$ which makes the spectral density very sensitive to the interatomic distance, and ii) the dynamical part which is the Fourier transform of a correlation function involving the Legendre polynomial. We shall denote this Fourier transform by $\tilde{J}(\omega)$ (we shall dub this quantity "normalized spectral density"). For calculating the relevant longitudinal relaxation rate, one has to take into account the transition probabilities in the energy diagram of a two-spin system. In the expression below, the first term corresponds to the double quantum transition (DQ), the second term to single quantum transitions (1Q) and the third term to the zero quantum transition (ZQ).

$$
R_{1, d}^{A}=\left(K_{d} / r_{A B}^{6}\right)\left[6 \tilde{J}_{d}\left(\omega_{A}+\omega_{B}\right)+3 \tilde{J}_{d}\left(\omega_{A}\right)+\tilde{J}_{d}\left(\omega_{A}-\omega_{B}\right)\right]
$$

\subsection{Chemical shift anisotropy (csa)}

This mechanism arises from the asymmetry of the shielding tensor. Let us recall that the chemical shift in NMR has its origin in the screening (shielding) of the static magnetic field $B_{0}$ by the electronic distribution at the level of the considered nucleus. This effect proceeds in fact from a tensorial quantity and the screening coefficient which defines the chemical shift in the liquid phase is just one third of the (Cartesian) tensor trace. Now, as far as relaxation is concerned, we have to consider the whole tensor and, more precisely, the system of its principal molecular axes $(x, y, z)$ in which the tensor is diagonal. Let us denote by $Z$ the direction of $B_{0}$.

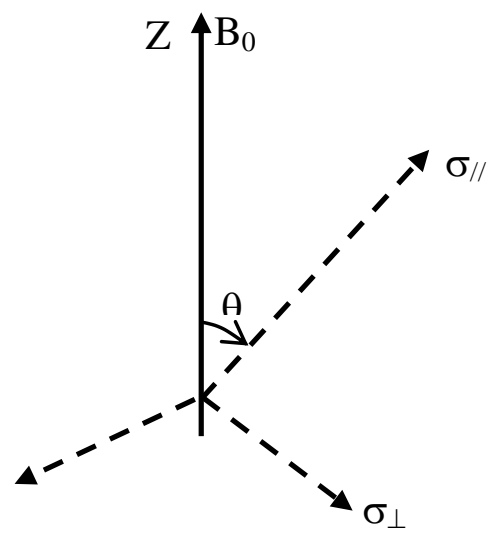

Figure 2. Principal axis system of a shielding tensor assumed to be of axial symmetry. 
The static magnetic field sensed by the considered nucleus is given by $B_{0}\left(1-\sigma_{Z}\right)$, where $\sigma_{Z Z}$ is the shielding for a given molecular orientation (figure 2). Of course, $\sigma_{Z Z}$ which is defined in the laboratory frame must be expressed as a function of the shielding tensor in a molecular frame where it is diagonal (the principal axis system). Since the latter rotates with respect to the laboratory frame, it is conceivable that the shielding effect can constitute a relaxation mechanism. For simplicity we shall assume a shielding tensor of axial symmetry (figure 2 ). $\sigma_{Z Z}$ can be expressed as

$$
\sigma_{Z Z}=\frac{\sigma}{3}+\frac{\Delta \sigma}{3}\left(3 \cos ^{2} \theta-1\right)
$$

where we have introduced the isotropic shielding coefficient (responsible for the chemical shift in liquid phase, hence the appellation "isotropic chemical shift")

$$
\sigma=\sigma_{/ /}+2 \sigma_{\perp}
$$

and the anisotropy of the shielding tensor (usually called "chemical shift anisotropy")

$$
\Delta \sigma=\sigma_{/ /}-\sigma_{\perp}
$$

The time dependence result evidently from the angle $\theta$ and, considering from a quantum mechanical point of view the interaction between a magnetic moment and the static magnetic field $B_{0}$ (Zeeman term), we can invoke a local field of the form

$$
b(t) \propto B_{0} \Delta \sigma\left[3 \cos ^{2} \theta(t)-1\right]
$$

Thus, the relevant spectral density, here equal to the longitudinal relaxation rate, is given by

$$
J_{c s a}(\omega)=R_{1, c s a}=(1 / 15)\left(\gamma B_{0}\right)^{2}(\Delta \sigma)^{2} \widetilde{J}_{c s a}(\omega)
$$

$\tilde{J}(\omega)$ refers to the motion of the tensor symmetry axis. An immediate consequence of (12) is the proportionality of the csa contribution to the square of the static magnetic field 
value, meaning that it can be safely neglected, in the case of carbon-13, up to $4.7 \mathrm{~T}$ (proton resonance frequency of $200 \mathrm{MHz}$ ). Another important feature of csa relaxation is its dependence with respect to the anisotropy $\Delta \sigma$ (expressed here in ppm), which is weak for aliphatic carbons but of the order of 150-200 ppm for ethylenic, aromatic or carbonyl carbons.

In the general case, when the shielding tensor is not of axial symmetry, in place of its diagonal elements $\sigma_{x x}, \sigma_{y y}$ and $\sigma_{z z}$ with $\left|\sigma_{z z}\right| \geq\left|\sigma_{y y}\right| \geq\left|\sigma_{x x}\right|$, the following parameters are generally used : $\sigma_{i s o}=\left(\sigma_{x x}+\sigma_{y y}+\sigma_{z z}\right) / 3, \Delta \sigma=\sigma_{z z}-\left(\sigma_{x x}+\sigma_{y y}\right) / 2$ and the asymmetry parameter $\eta=\left(\sigma_{x x}-\sigma_{y y}\right) / \sigma_{z z}=(3 / 2)\left(\sigma_{x x}-\sigma_{y y}\right) / \Delta \sigma$. The second form of $\eta$ arises from the fact that, as far as relaxation is concerned, the shielding tensor can be defined with respect to any time-independent reference (which therefore will not act as a relaxation mechanism). $\sigma_{i s o}$ is such a reference and will be taken as zero, hence the second form of $\eta$. Now, if the molecular reorientational motion is isotropic, (12) is transformed into

$$
R_{1, c s a}=(1 / 15)\left(\gamma B_{0}\right)^{2}\left(1+\eta^{2} / 3\right)(\Delta \sigma)^{2} \tilde{J}_{c s a}(\omega)
$$

\section{Cross-relaxation (and cross-correlation). Solomon equations}

\subsection{Simple Solomon equations ${ }^{7}$}

Whenever the system is no longer constituted by single non-interacting spins, the simple Bloch equation (2) must be complted by additional coupling terms. Let us consider the dipolar interaction between two spins $1 / 2 A$ and $B$. This interaction is responsible for a biexponential evolution of their polarization which is accounted for by two simultaneous differential equations called Solomon equations 


$$
\begin{aligned}
& \frac{d I_{z}^{A}}{d t}=-R_{1}^{A}\left(I_{z}^{A}-I_{e q}^{A}\right)-\sigma_{A B}\left(I_{z}^{B}-I_{e q}^{B}\right) \\
& \frac{d I_{z}^{B}}{d t}=-R_{1}^{B}\left(I_{z}^{B}-I_{e q}^{B}\right)-\sigma_{A B}\left(I_{z}^{A}-I_{e q}^{A}\right)
\end{aligned}
$$

The coupling term, traditionally denoted by $\sigma_{A B}$ (which has however nothing to do with the screening coefficient of section 2.2), is the so-called cross-relaxation rate and is a relaxation parameters which depends exclusively on the dipolar interaction between nuclei $A$ and $B$, contrary to auto-relaxation rates which are compounds of several contributions. For instance, if $A$ is a carbon-13, the auto-relaxation rate can always be written as

$$
R_{1}^{A}=R_{1, \text { dip }}^{A}+R_{1, \text { csa }}^{A}+R_{1, \text { others }}^{A}
$$

In (15), $R_{1, \text { others }}^{A}$ encompasses all secondary interactions which are not included in the first two terms (for instance the interaction with an unpaired electron, the spin-rotation interaction...). By contrast, the expression of the cross-relaxation rate is simply

$$
\sigma_{A B}=\left(K_{d} / r_{A B}^{6}\right)\left[6 \tilde{J}_{d}\left(\omega_{A}+\omega_{B}\right)-\tilde{J}_{d}\left(\omega_{A}-\omega_{B}\right)\right]
$$

This is the beauty of this quantity which provides specifically a direct geometrical information $\left(1 / r_{A B}^{6}\right)$ provided that the dynamical part of (16) can be inferred from appropriate experimental determinations. This cross-relaxation rate, first discovered by Overhauser in 1953 about proton-electron dipolar interactions ${ }^{8}$, led to the so-called nuclear Overhauser effect (nOe) in the case of nucleus-nucleus dipolar interactions, and has found tremendous applications in $\mathrm{NMR}^{2}$. As a matter of fact, this review is purposely limited to the determination of proton-carbon-13 cross-relaxation rates in small or medium-size molecules and to their interpretation.

The simplest way to measure the proton-carbon-13 cross-relaxation rate is to saturate the proton transitions by means of decoupling procedures, which are normally used to remove the effect of proton-carbon $\mathrm{J}$ couplings from carbon-13 spectra, thus leading to a single carbon peak (provided there exists no J coupling with other nuclei). Referring to the first 
of equations (14) and supposing that $A$ is the carbon-13 nucleus, $B$ being the proton coupled by dipolar interaction to $A$, one has

$$
\frac{d I_{z}^{A}}{d t}=-R_{1}^{A}\left(I_{z}^{A}-I_{e q}^{A}\right)+\sigma_{A B} I_{e q}^{B}
$$

Assuming further that decoupling has been turned on a long time ago before the carbon-13 measurement so that a steady state is reached, one ends up with a steady state carbon polarization, $I_{s t}^{A}$, obtained by setting $\frac{d I_{z}^{A}}{d t}$ to zero:

$$
I_{s t}^{A}=\left(1+\frac{\gamma_{B}}{\gamma_{A}} \frac{\sigma_{A B}}{R_{1}^{A}}\right) I_{e q}^{A}
$$

Now, it can be seen from equation (17) that a simple relaxation measurement under decoupling conditions yields directly $R_{1}^{A}$ whereas $I_{e q}^{A}$ can be measured by a standard experiment described in section 5. One then arrives to the so-called nOe factor $\eta$ given by

$$
\eta=\frac{I_{s t}^{A}-I_{e q}^{A}}{I_{e q}^{A}}=\frac{\gamma_{B}}{\gamma_{A}} \frac{\sigma_{A B}}{R_{1}^{A}}
$$

It can be noticed that the maximum nOe factor ( 2 when $A$ is a carbon- 13 and $B$ a proton) is reached under extreme narrowing (see section 6) conditions and if $R_{1}^{A}$ arises exclusively from the $A-B$ dipolar interaction. On the other hand, the cross-relaxation rate $\sigma_{A B}$ is easily deduced from the nOe factor and from the $A$ specific relaxation rate

$$
\sigma_{A B}=\frac{\gamma_{A}}{\gamma_{B}} \eta R_{1}^{A}
$$

In spite of the apparent simplicity of the method, its drawback comes from the fact that a two-spin system has been assumed. It provides merely global information spanning all protons prone two interact by dipolar coupling with the considered carbon. Selective information requires pulsed experiments stemming from the general solution of equations (14) given below. 
For this purpose, let us define $A$ by $A=I_{z}^{A}-I_{e q}^{A}$ and $B$ by $B=I_{z}^{B}-I_{e q}^{B}$, with $A(0)=K_{A}$, $B(0)=K_{B}$. Solomon equations can then be written as

$$
\begin{aligned}
& \frac{d A}{d t}=-R_{1}^{A} A-\sigma_{A B} B \\
& \frac{d B}{d t}=-R_{1}^{B} B-\sigma_{A B} A
\end{aligned}
$$

Their solution is as follows

$$
\begin{aligned}
& A=a_{+} e^{\lambda_{+} t}+a_{-} e^{\lambda_{-} t} \\
& B=b_{+} e^{\lambda_{+} t}+b_{-} e^{\lambda_{-} t}
\end{aligned}
$$

$\lambda_{+}$and $\lambda_{-}$are both negative and are expressed as

$$
\lambda_{ \pm}=-\frac{R_{1}^{A}+R_{1}^{B}}{2} \pm \frac{1}{2} \sqrt{\left(R_{1}^{A}-R_{1}^{B}\right)^{2}+4 \sigma_{A B}^{2}}
$$

The coefficients $\left(a_{+}, a_{-}, b_{+}, b_{-}\right.$depend on all relaxation parameters and, of course, on the initial conditions). One has

$$
\begin{aligned}
& b_{+}=-\frac{\sigma_{A B}}{\lambda_{+}+R_{1}^{B}} a_{+} \\
& b_{-}=-\frac{\sigma_{A B}}{\lambda_{-}+R_{1}^{B}} a_{-}
\end{aligned}
$$

and

$$
\begin{aligned}
& a_{+}=\frac{K_{A} /\left(\lambda_{-}+R_{1}^{B}\right)+K_{B} / \sigma_{A B}}{1 /\left(\lambda_{-}+R_{1}^{B}\right)-1 /\left(\lambda_{+}+R_{1}^{B}\right)} \\
& a_{-}=\frac{K_{A} /\left(\lambda_{+}+R_{1}^{B}\right)+K_{B} / \sigma_{A B}}{1 /\left(\lambda_{+}+R_{1}^{B}\right)-1 /\left(\lambda_{-}+R_{1}^{B}\right)}
\end{aligned}
$$

The crucial point arises from $\lambda_{+}$which may become very small, thus leading to the little known property of long lived states. For this purpose let us assume that $R_{1}^{B}$, in our case the proton longitudinal relaxation rate, is much greater than $R_{1}^{A}$ and $\sigma_{A B}$, a common situation which, for $\lambda_{+}$, leads to a quantity which can be very small 


$$
\lambda_{+} \cong-R_{1}^{A}+\frac{\sigma_{A B}^{2}}{R_{1}^{B}}
$$

$\lambda_{-} \cong-R_{1}^{B}$ is large and governs the first part of the evolution. Conversely, when the corresponding term in (22) has almost completely decayed to zero, it essentially remains the term involving $\lambda_{+}$, which generates a long lasting signal. The amplitude of the latter (and therefore the possibility of its detection) depends on initial conditions via the coefficient $a_{+}$.

\subsection{Longitudinal spin order. Extended Solomon equations}

So far, we have not considered the so-called longitudinal two-spin order, represented by the product operator ${ }^{9} 2 I_{z}^{A} I_{z}^{B}$, a quantity related to the polarization of nuclei $A$ and $B$. This spin state can be created in different ways. The easiest way is probably to let the system evolve under the sole $\mathrm{J}_{\mathrm{AB}}$ coupling so as to obtain an antiphase doublet, for instance the $B$ antiphase doublet represented by $2 I_{z}^{A} I_{x}^{B}$ (corresponding to the two proton carbon-13 satellites in an antiphase configuration). Applying selectively on $B$ a $(\pi / 2)_{y}$ pulse transforms the latter spin state into $2 I_{z}^{A} I_{z}^{B}$ (here $x, y, z$ refer to the rotating frame). It turns out that the longitudinal order can also be created by relaxation. This is due to a coupling term which acts in the same way as the cross-relaxation rate but, this time, between $A$ and $B$ polarization and the longitudinal order. This coupling term is called cross-correlation rate $^{10}$ because it involves two relaxation mechanisms, namely the $A-B$ dipolar interaction and the csa mechanism at $A$ and $B$. In fact, two such cross-correlation terms are prone to interfere with the evolution of $I_{z}^{A}$ and $I_{z}^{B}$. They will be denoted as $\sigma_{c s a(A), d}$ and $\sigma_{c s a(B), d}$; they complicate somewhat equations (21) and give rise to the extended Solomon equations which can be written as (with $A B=2 I_{z}^{A} I_{z}^{B}$ and recognizing that the longitudinal 
order is zero at equilibrium)

$$
\begin{aligned}
& \frac{d A}{d t}=-R_{1}^{A} A-\sigma_{A B} B-\sigma_{c s a(A), d} A B \\
& \frac{d B}{d t}=-R_{1}^{B} B-\sigma_{A B} A-\sigma_{c s a(B), d} A B \\
& \frac{d A B}{d t}=-R_{1}^{A B} A B-\sigma_{c s a(A), d} A-\sigma_{c s a(B), d} B
\end{aligned}
$$

$R_{1}^{A B}$ is the longitudinal order auto-relaxation which may depend on all relaxation mechanisms affecting the spin system. We give below the dipolar and csa contributions

$$
\begin{aligned}
& R_{1}^{A B}=\left(K_{d} / r_{A B}^{6}\right)\left[3 \tilde{J}_{d}\left(\omega_{A}\right)+3 \tilde{J}_{d}\left(\omega_{B}\right)\right]-(1 / 15)\left(\gamma_{A} B_{0}\right)^{2}\left(\Delta \sigma_{A}\right)^{2} \tilde{J}_{c s a(A)}\left(\omega_{A}\right) \\
& -(1 / 15)\left(\gamma_{B} B_{0}\right)^{2}\left(\Delta \sigma_{B}\right)^{2} \tilde{J}_{c s a(B)}\left(\omega_{B}\right)
\end{aligned}
$$

Conversely, the cross-correlation rates depend solely on the csa mechanism and on the dipolar interaction which is of prime importance here. It arises in fact from correlation functions of the form $\overline{b^{\prime}(t) b(0)}$ where $b^{\prime}(t)$ refers to the csa mechanism whereas $b(t)$ refers to the dipolar interaction. One has

$$
\sigma_{c s a(A), d}=-\left(\frac{1}{5}\right) \sqrt{\frac{2}{3}}\left(\gamma_{A} B_{0}\right)\left(\Delta \sigma_{A}\right)\left(\frac{\mu_{0}}{4 \pi}\right)\left(\frac{\gamma_{A} \gamma_{B} \hbar}{r_{A B}^{3}}\right) \tilde{J}_{c s a(A), d}\left(\omega_{A}\right)
$$

The various symbols have the same meaning as before while the spectral density $\tilde{J}_{c s a(A), d}\left(\omega_{A}\right)$ will be discussed in section 4 . For the moment, let us state that these cross-correlation rates can play a role only if the csa mechanism is important (i.e. for non aliphatic carbons but certainly not for protons) and if measurements are performed at high field (due to the term $\gamma_{A} B_{0}$ in (29)). This means that, if $A$ is a carbon-13 and $B$ a proton, $\sigma_{c s a(B), d}$ can be safely neglected in (27). Nevertheless, the exploitation of (27), which involves three simultaneous differential equations, relies necessarily on numerical procedures. In order to convince the reader that extended Solomon equations are not purely theoretical, we provide in figure 3 an illustrative example showing the longitudinal order build-up. 


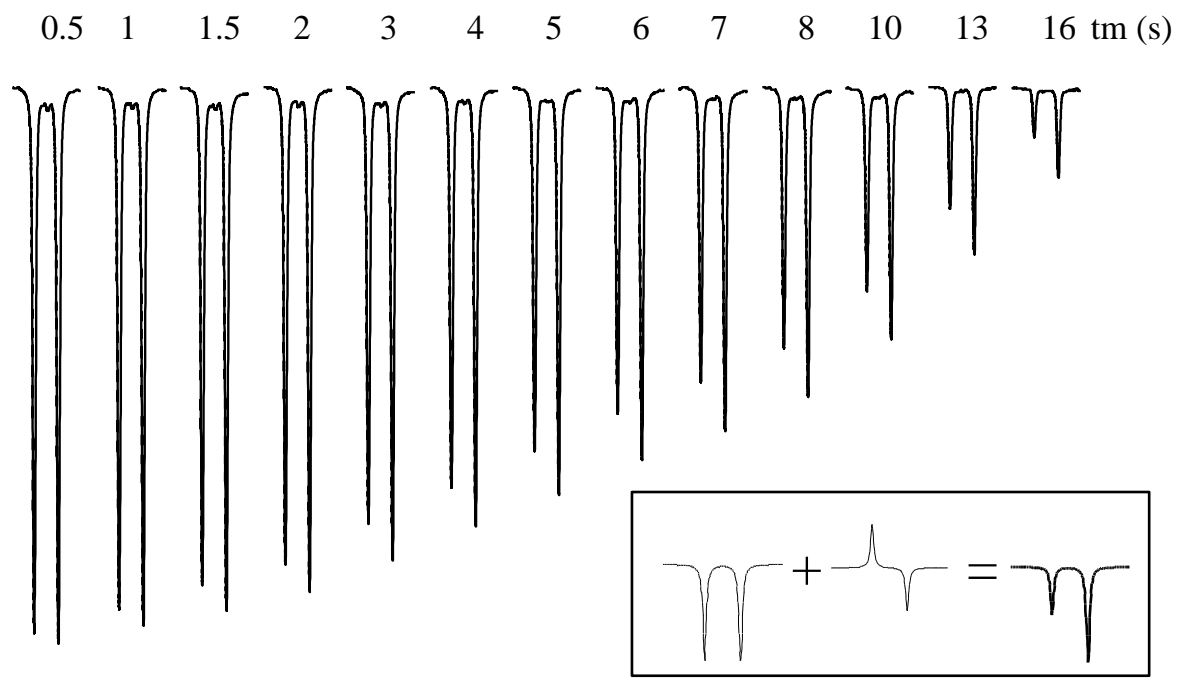

Figure 3. Creation of the longitudinal order by cross-correlation as a function of the mixing time $\mathrm{t}_{\mathrm{m}}$ which follows the inversion of a carbon-13 doublet (due to a $\mathrm{J}$ coupling with a bonded proton). The read-pulse transforms the longitudinal polarization into an inphase doublet and the longitudinal order into an antiphase doublet. The superposition of these two doublets leads to the observation of an asymmetric doublet.

Finally, it can be noted that there also exist dipolar-dipolar cross-correlation rates which involve two different dipolar interactions. These quantities may play a role, for instance, in the carbon-13 longitudinal relaxation of ${\mathrm{a} \mathrm{CH}_{2} \text { grouping }}^{11,12}$. Due to the complexity of the relevant theory and to their marginal effect under proton decoupling conditions, they will be disregarded in the following.

\section{Spectral densities}

As seen from the above theoretical developments, accessing geometrical (and stereochemical) information implies at least an estimation of the dynamical part of the various relaxation parameters. The latter is represented by spectral densities which rest on the calculation of the Fourier transform of auto- or cross-correlation functions. These calculations require necessarily a model for describing molecular reorientation (overall and internal) since this review is limited to intra-molecular relaxation mechanisms. 
Conversely, inter-molecular dipolar interactions would imply complex models involving, among other things, translational diffusion processes ${ }^{13}$. This aspect of nuclear spin relaxation will not be considered here.

\subsection{Isotropic tumbling of a rigid molecule}

Small-step rotational diffusion is the model universally used for characterizing the overall molecular reorientation. If the molecule is of spherical symmetry (or approximately; this is generally the case for molecules of important size), a single rotational diffusion coefficient is needed and the molecular tumbling is said isotropic. According to this model, correlation functions obey a diffusion type equation and we can write

$$
\overline{b(t) b(0)}=\overline{b^{2}} e^{-t / \tau_{c}}
$$

$\tau_{c}$ is called the correlation time and is equal to $1 / 6 D_{r}$ where $D_{r}$ is the rotational diffusion coefficient. For a simple Brownian motion, $D_{r}$ can be expressed as a function of the radius $a$ of the molecule (assumed to be spherical) and of the solvent viscosity $\eta$. This is the well-known Stokes-Einstein equation

$$
D_{r}=\frac{k_{B} T}{8 \pi a^{3} \eta}
$$

where $k_{B}$ is the Boltzmann constant and $T$ the absolute temperature.

Qualitatively, $\tau_{c}$ can be viewed as the time necessary for a reorientation by one radian. $\tau_{c}$ is very weak $\left(10^{-11}-10^{-12} \mathrm{~s}\right)$ for small size molecules in non viscous solvents. Conversely, for large molecules (such as proteins in aqueous solution), it can reach much more important values $\left(10^{-9} \mathrm{~s}\right.$ or higher). All (normalized) auto-correlation spectral densities have the same expressions since, in the molecule, all directions are equivalent

$$
\tilde{J}(\omega)=\frac{2 \tau_{c}}{1+\omega^{2} \tau_{c}^{2}}
$$


(32) is as well valid for dipolar or csa autocorrelation spectral densities. As $\omega$ is an NMR frequency, thus smaller than $6.310^{9} \mathrm{rad} \mathrm{s}^{-1}, \tilde{J}(\omega)$ is generally frequency independent for small or medium size molecules $\left(\omega^{2} \tau_{c}^{2}<<1\right)$ and simply equal to $2 \tau_{c}$. Such a situation is called extreme narrowing. However, depending on the medium or on possible molecular associations, extreme narrowing conditions may no longer prevail and $\tau_{c}$ can be easily deduced from the evolution of relaxation parameters with the measurement frequency. This method, called relaxometry, can be employed whenever $\tau_{c}$ becomes larger than $10^{-10}$ s (see figure 4).

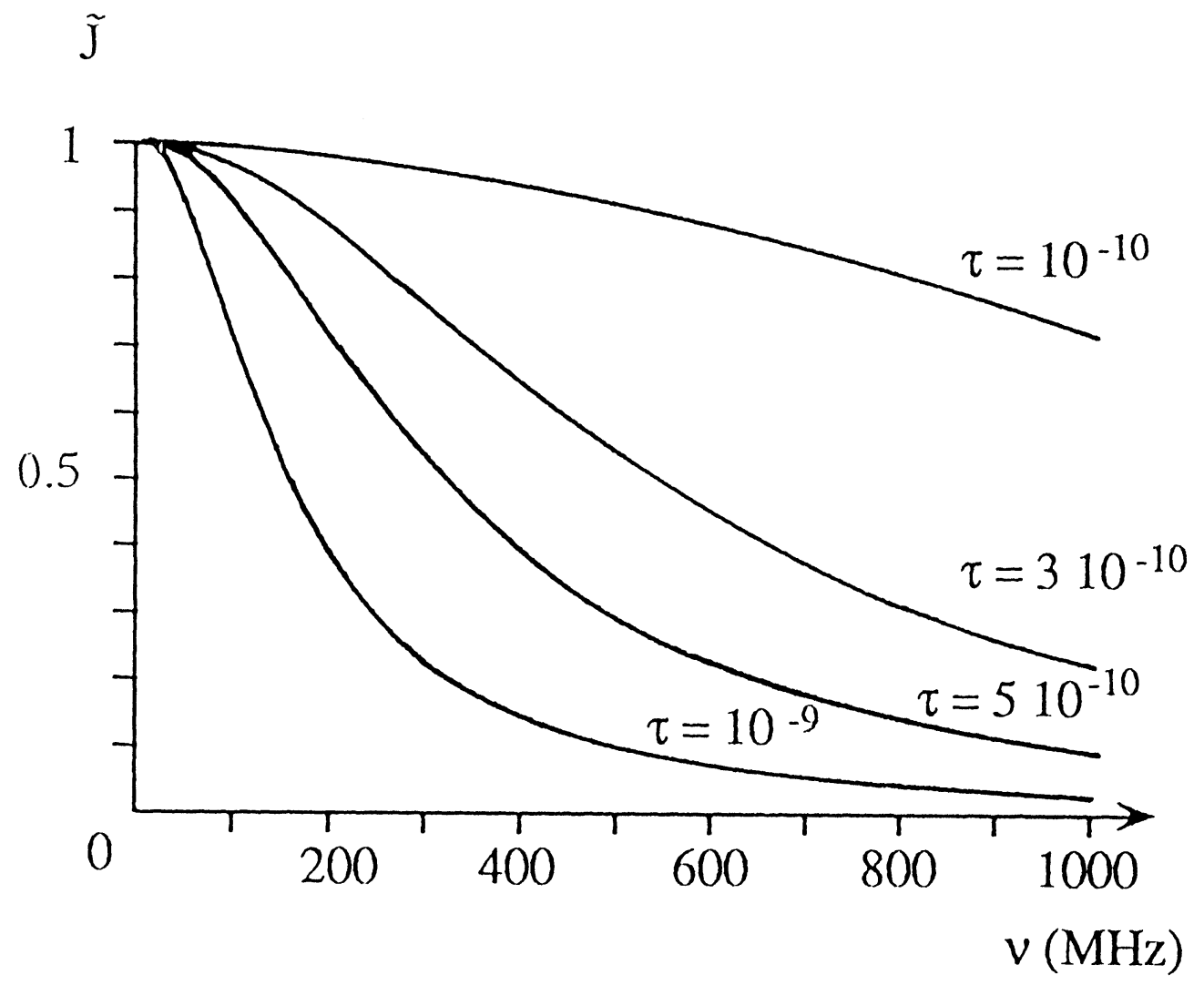

Figure 4. Normalized spectral densities as a function of $v=\omega / 2 \pi$ for different values of the correlation time $\tau$ (in s). They all start from 1 just to highlight their dependence with respect to the measurement frequency.

Concerning the cross-correlation spectral densities introduced in (29), they are of the same 
form as (32) with a geometrical factor depending on the angle $\theta_{d, c s a}$ between the two relaxation vectors: the vector joining the two nuclei for the dipolar interaction, and the largest shielding principal axis (or the symmetry axis of the shielding tensor if it is of axial symmetry). One has

$$
\tilde{J}_{c s a(A), d}\left(\omega_{A}\right)=(1 / 2)\left(3 \cos ^{2} \theta_{d, c s a}-1\right)\left(\frac{2 \tau_{c}}{1+\omega_{A}^{2} \tau_{c}^{2}}\right)
$$

\subsection{Anisotropic tumbling of a rigid molecule}

If the considered molecule cannot be assimilated to a sphere, one has to take into account a rotational diffusion tensor, the principal axes of which coincide, to a first approximation, with the principal axes of the molecular inertial tensor. In that case, three different rotational diffusion coefficients are needed ${ }^{14}$. They will be denoted as $D_{X}, D_{Y}, D_{Z}$ and describe the reorientation about the principal axes of the rotational diffusion tensor. They lead to unwieldy expressions even for auto-correlation spectral densities, which can be somewhat simplified if the considered interaction can be approximated by a tensor of axial symmetry, allowing us to define two polar angles $\theta$ and $\varphi$ describing the orientation of the relaxation vector (the symmetry axis of the considered interaction) in the $(X, Y, Z)$ molecular frame (see figure 5). As the tensor associated with dipolar interactions is necessarily of axial symmetry (the relaxation vector being the inter-nuclear vector) and because this review deals essentially with dipolar interactions, we shall limit ourselves to auto-correlation spectral densities in the case of an axially symmetric tensor.

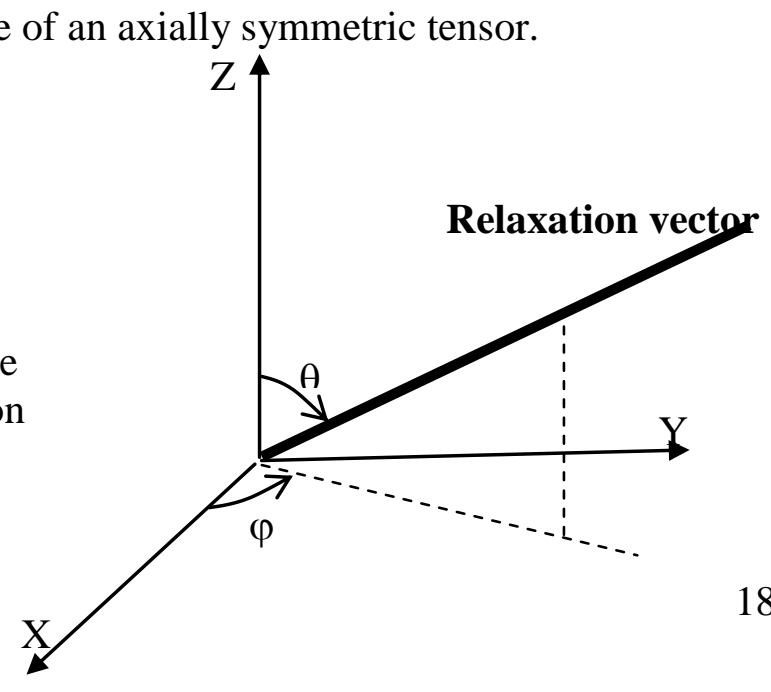

Figure 5. Definition of the polar angles in the principal axis system of the diffusion-rotation tensor. 
From the pioneering works of Woessner ${ }^{15}$, Huntress ${ }^{16}$ and Hubbard ${ }^{17}$, we can derive the following formulae ${ }^{18}$

$$
\widetilde{J}_{\text {auto }}(\omega)=\sum_{k=-2}^{2} a_{k} \frac{\left(2 / \lambda_{k}\right)}{1+\omega^{2} / \lambda_{k}^{2}}
$$

with

$$
\begin{aligned}
& \lambda_{0}=4 D_{Z}+2 D_{+} \\
& \lambda_{ \pm 1}=D_{Z}+5 D_{+} \pm 3 D_{-} \\
& \lambda_{ \pm 2}=2 D_{Z}+4 D_{+} \pm\left[4\left(D_{Z}-D_{+}\right)^{2}+12 D_{-}^{2}\right]^{1 / 2} \\
& D_{ \pm}=\left(D_{X} \pm D_{Y}\right) / 2
\end{aligned}
$$

and

$$
\begin{aligned}
& a_{0}=(3 / 4) \sin ^{4} \theta \sin ^{2} 2 \varphi \\
& a_{1}=(3 / 4) \sin ^{2} 2 \theta \sin ^{2} \varphi \\
& a_{-1}=(3 / 4) \sin ^{2} 2 \theta \cos ^{2} \varphi \\
& a_{ \pm 2}=\frac{1}{12 D_{-}^{2}+d_{ \pm}^{2}}\left\{\left(9 D_{-}^{2}\right) \sin ^{4} \theta \cos ^{2} 2 \varphi-\left(3 D_{-} d_{ \pm}\right)\left[\sin ^{2} \theta \cos 2 \varphi\left(3 \cos ^{2} \theta-1\right)\right]+\right. \\
& \left.\frac{d_{ \pm}^{2}}{4}\left(3 \cos ^{2} \theta-1\right)^{2}\right\} \\
& d_{ \pm}=2\left(D_{Z}-D_{+}\right) \mp\left[4\left(D_{Z}-D_{+}\right)^{2}+12 D_{-}^{2}\right]^{1 / 2}
\end{aligned}
$$

Fortunately, in the case of a rotational diffusion tensor with axial symmetry (such molecules are denoted "symmetric top"), some simplification occurs. Let us introduce new notations: $D_{/ /}=D_{Z}$ and $D_{\perp}=D_{X}=D_{Y}$. Furthermore, we shall define effective correlation times:

$$
\begin{aligned}
& 1 / \tau_{0}=6 D_{\perp} \\
& 1 / \tau_{1}=5 D_{\perp}+D_{/ \prime} \\
& 1 / \tau_{2}=2 D_{\perp}+4 D_{/ /}
\end{aligned}
$$

With these notations, one has

$$
\tilde{J}_{\text {auto }}(\omega)=\frac{1}{4}\left(3 \cos ^{2} \theta-1\right)^{2} \frac{2 \tau_{0}}{1+\omega^{2} \tau_{0}^{2}}+\frac{3}{4} \sin ^{2} 2 \theta \frac{2 \tau_{1}}{1+\omega^{2} \tau_{1}^{2}}+\frac{3}{4} \sin ^{4} \theta \frac{2 \tau_{2}}{1+\omega^{2} \tau_{2}^{2}}
$$

It can be noticed that at least two independent relaxation parameters in the symmetric top case, and three in the case of fully anisotropic diffusion rotation are necessary for deriving the rotation diffusion coefficients, provided that the relevant structural parameters are known and that the orientation of the rotational diffusion tensor has been deduced from symmetry 
considerations or from the inertial tensor.

\subsection{Local motions. The model free approach}

Molecular internal motions are prone to affect relaxation parameters. A first approximation is to assume that they participate in the rotational diffusion anisotropy and to use the formulation of the preceding section. Indeed, for treating the internal rotation of a methyl grouping, one can use ${ }^{19}$ an expression very close to (38). It can also be assumed that the overall tumbling and internal motions are independent so that it is possible to devise models which account for the superposition of these two types of motion. These models ${ }^{20-24}$, depending on a number of parameters which may exceed the number of observables, will not be further detailed. We shall rather focus on the popular "model free approach", also called the Lipari-Szabo model ${ }^{25}$ (the relevant expressions were actually derived earlier by Wennerström et $a l_{.}{ }^{26}$ ) which treats pragmatically the superposition of isotropic overall tumbling and local internal motions with a reasonable number of parameters. This approach is based on the fact that internal motions, considered globally, are represented by a correlation time $\tau_{f}$ ( $f$ for fast motions assumed to lead to extreme narrowing conditions) and an order parameter $S$ which reflects their anisotropic character or rather their orientational restriction. $S$ has the same meaning than in liquid crystals, reflecting a partial orientation with respect to a given local director. It is an empirical parameter defined as the mean of $(1 / 2)\left(3 \cos ^{2} \theta-1\right), \theta$ being the angle between the relaxation vector and the director. It ranges from -0.5 to 1 , the latter value corresponding to a uniform orientation while $S=0$ indicates a pure random orientation (that is no preferred direction). $S=-0.5$ indicates that all relaxation vectors are perpendicular to the director. The overall tumbling is characterized by a correlation time $\tau_{s}(s$ for slow, this approach being really interesting when the overall tumbling is outside extreme narrowing conditions). The relevant auto-correlation spectral density, suitable for dipolar 
interactions ( $S$ would be the order parameter of the internuclear direction), is of the form

$$
\tilde{J}_{\text {auto }}^{\text {dip }}(\omega)=\left(1-S^{2}\right)\left(2 \tau_{f}\right)+S^{2} \frac{2 \tau_{s}}{1+\omega^{2} \tau_{s}^{2}}
$$

$S, \tau_{f}$ and $\tau_{s}$ have to be deduced from experimental data. It can be noted that $\frac{2 \tau_{s}}{1+\omega^{2} \tau_{s}^{2}}$ in (39) can be substituted by (34) or (38) if the overall tumbling is anisotropic. (39) would also be suitable for the csa contribution to relaxation rate provided that the csa tensor is of axial symmetry and that one defines a specific order parameter for the relevant symmetry axis. Let $\alpha$ the angle between the dipolar internuclear direction and the csa symmetry axis. If (39) is associated with the dipolar interaction, the homologous csa spectral density can be written $\operatorname{as}^{27}$

$$
\tilde{J}_{\text {auto }}^{c s a}(\omega)=\left[1-(1 / 4)\left(3 \cos ^{2} \alpha-1\right)^{2} S^{2}\right]\left(2 \tau_{f}\right)+(1 / 4)\left(3 \cos ^{2} \alpha-1\right)^{2} S^{2} \frac{2 \tau_{s}}{1+\omega^{2} \tau_{s}^{2}}
$$

Similarly $^{27}$, the dipolar-csa cross-correlation spectral density can be expressed as follows

$$
\widetilde{J}_{\text {cross }}^{\text {dip, csa }}(\omega)=(1 / 2)\left(3 \cos ^{2} \alpha-1\right)\left[\left(1-S^{2}\right)\left(2 \tau_{f}\right)+S^{2} \frac{2 \tau_{s}}{1+\omega^{2} \tau_{s}^{2}}\right]
$$

The Lipari-Szabo approach has been essentially used in the study of large biomolecules ${ }^{21-24,28}$, less often for the medium size molecules as will be discussed in section 6 .

\section{Experimental procedures}

From now onward, we shall assume natural abundance for carbon isotopes, so that the signal we have to deal with arises from a single carbon-13 in the molecule under investigation. Moreover, most of the experiments described below imply, at one stage or another, proton decoupling for removing any multiplet structure (due to $\mathrm{J}$ couplings with protons) in such a way that every carbon gives rise to a singlet (except J couplings with other nuclei such as ${ }^{19} \mathrm{~F}$, $\left.{ }^{31} \mathrm{P} . ..\right)$. Another consequence of proton decoupling is, in principle, the destruction of proton 
magnetization so that the Solomon equation concerning carbon-13 (see (14)) reduces to

$$
\frac{d I_{z}^{A}}{d t}=-R_{1}^{A}\left(I_{z}^{A}-I_{s t}^{A}\right)
$$

where $I_{s t}^{A}$ is given by (18). This is an important property meaning that, under continuous proton decoupling, carbon-13 longitudinal relaxation is mono-exponential. Very often (either in exploiting the nOe factor or the Solomon equations), the knowledge of the carbon-13 longitudinal relaxation time will be required. The forthcoming section is thus devoted to its measurement.

\subsection{Measurement of ${ }^{13} \mathrm{C}$ longitudinal relaxation time}

As explained above, these experiments have to be performed under continuous proton decoupling. The usual method is inversion-recovery ${ }^{29}$ (figure 6) which would require, between consecutive experiments or consecutive scans, a waiting time of $5 T_{1}$ unless one has recourse to a variant dubbed "Fast Inversion-Recovery"30.

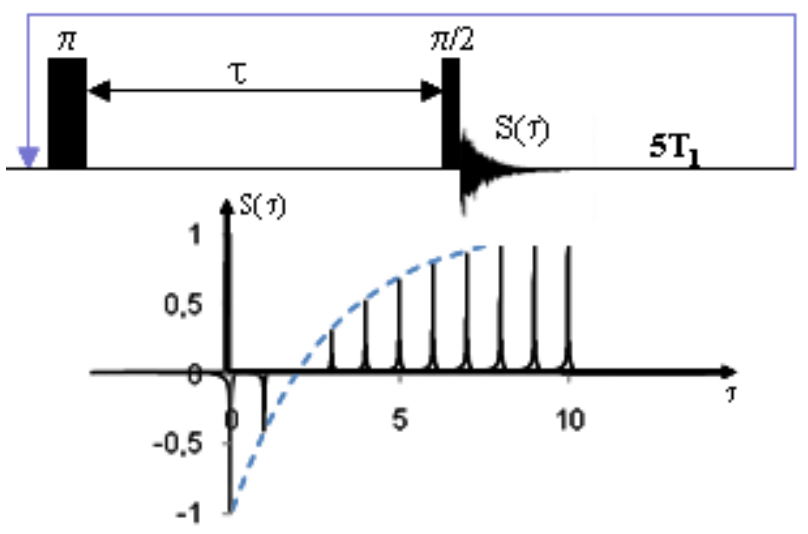

Figure 6. The inversion-recovery experiment. The dynamic range is twice the equilibrium magnetization

Saturation-recovery $^{31}$ (figure 7) is an interesting alternative in the case of very long longitudinal relaxation times or when a proper inverting pulse is not available. It makes use of a saturation pulse (i.e. capable of destroying the whole carbon-13 magnetization) in place of 
the $\pi$ pulse of the inversion-recovery experiment. Since magnetization starts always from zero, no waiting time is needed. The only drawback is a dynamic range half the one of the inversion-recovery experiment.

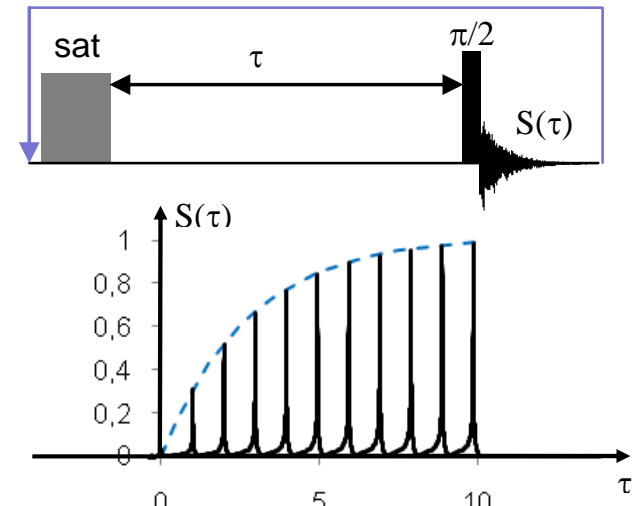

Figure 7. The saturation-recovery experiment. The dynamic range is equal to the equilibrium magnetization

For both experiments and according to (42), the experimental data can be adjusted according to

$$
S(\tau)=S_{e q}\left(1-k e^{-\tau / T_{1}}\right)
$$

$1<k<2$ for the inversion-recovery experiment ( $k$ would be equal to 2 for a perfect $180^{\circ}$ pulse and for a waiting time permitting full equilibrium recovery). $k$ is around 1 for the saturationrecovery experiment (it would be strictly equal to 1 in the case of a perfect saturation; in practice, it can be either larger or smaller).

\subsection{Measurement of the nOe factor}

The nOe factor provides a global information, that is the sum of cross-relaxation rates of all protons which can interact by dipolar coupling with the considered carbon. It is therefore very useful when the considered carbon is directly bound to one, two or three protons since the relevant dipolar interaction will overwhelm more remote interactions. As the $\mathrm{C}-\mathrm{H}$ distance is normally known, the dynamical part of the cross-relaxation rate is, in that case, readily available (see equation (16)) and can serve as a reference for further geometrical 
determinations. Now from equation (19), it can be seen that $I_{s t}^{A}$ and $I_{e q}^{A}$ have to be measured. In principle, the former is deduced from the usual proton-decoupled ${ }^{13} \mathrm{C}$ spectrum with a waiting time of five times the longest $T_{1}$ to restore equilibrium magnetization between scans. However, in experiments for which decoupling is substituted by a pulse train (this is the case in reverse two-dimensional experiments, mainly used for the study of large biomolecules), some caution must be exercised regarding the ability of actually saturating the proton spin system $^{32}$. For obtaining $I_{e q}^{A}$, it suffices to run the normal experiment but with decoupling switched on during the fid acquisition. This can be achieved ${ }^{33}$ that way because i) decoupling is operative instantaneously, ii) nOe does not affect transverse magnetization. However, since the proton spin state is modified during the pulse sequence (by gated decoupling), proton polarization also relaxes so that the recovery toward equilibrium needs a waiting time longer than $5 T_{1}$. Various simulations have shown that a waiting time of $10 T_{1}$ was safe in all circumstances $^{34}$. The procedures for measuring $I_{s t}^{A}$ and $I_{e q}^{A}$ are schematized in figure 8.
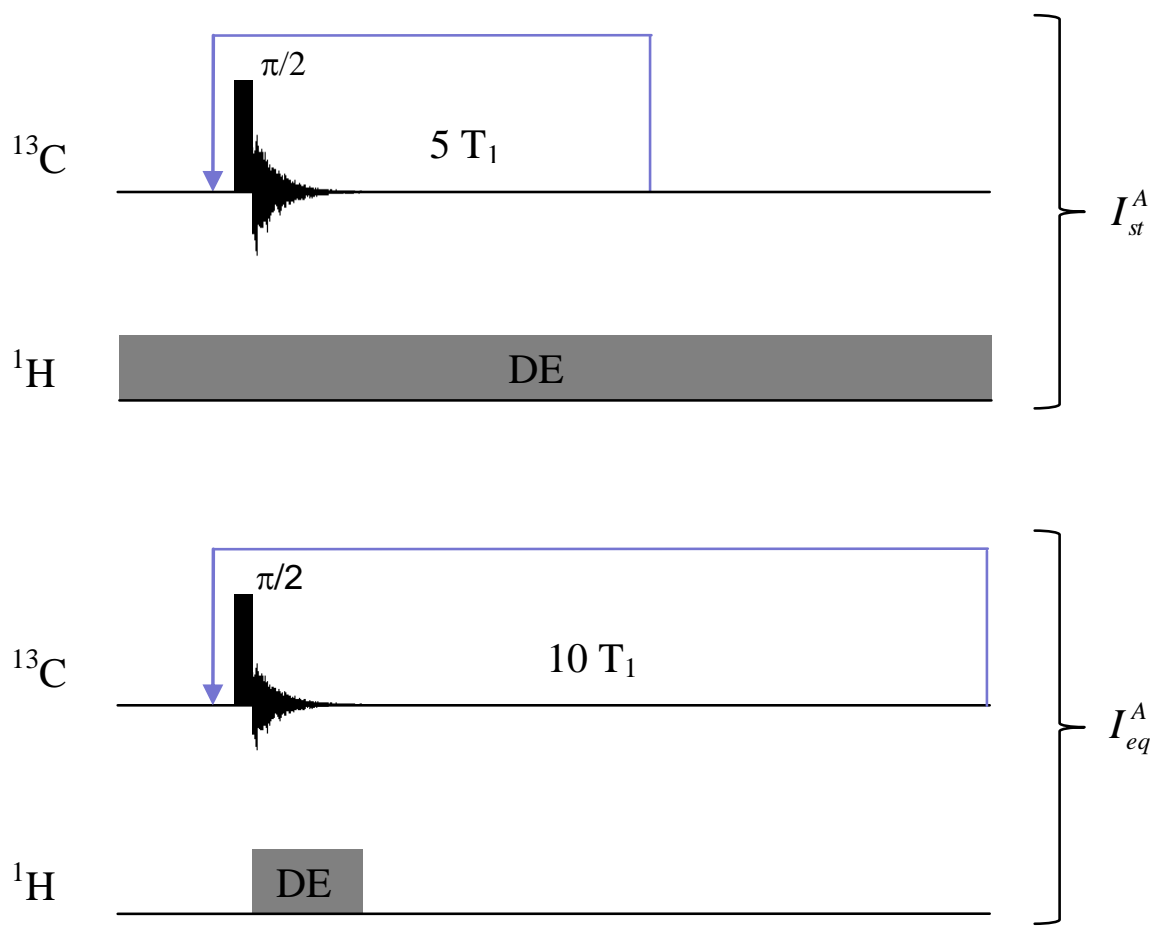

Figure 8. the two separate experiments for experimentally determining the nOe factor (see (19)). DE stands for decoupling. 
In the early days of carbon-13 spectroscopy, high power decoupling was used and nOe measurements had to be carried out in such a way that temperature was identical for both experiments (interleaved experiments with shift of the proton frequency). With modern spectrometers, such precautions are no longer necessary thanks to decoupling schemes which permit low power decoupling and thanks to accurate temperature control.

\subsection{The HOESY (Heteronuclear Overhauser Effect Spectroscopy) experiment}

Initially, this is a two-dimensional experiment which is supposed to be the heteronuclear homolog of the NOESY experiment. It thus involves ${ }^{1} \mathrm{H}$ and ${ }^{13} \mathrm{C}$ pulses as well as proton decoupling during ${ }^{13} \mathrm{C}$ signal acquisition. This experiment, proposed simultaneously by Rinaldi $^{35}$ on the one hand, and Yu and Levy ${ }^{36,37}$ on the other hand, provides only cross-peaks which indicate ${ }^{1} \mathrm{H}-{ }^{13} \mathrm{C}$ distance correlations. Evidently, at the onset, the method provides information about directly bonded ${ }^{1} \mathrm{H}-{ }^{13} \mathrm{C}$ nuclei, information which can be obtained through other more sensitive procedures. Its interest lies in other more remote correlations. Indeed, it has been mostly applied to intermolecular dipolar interactions ${ }^{36-43}$. As described later, we shall be rather interested here in intramolecular interactions associated with geometrical information not accessible by other correlation methods. The pulse sequences that we are using are shown in figure 9 and will be now commented. We have found it useful to slightly modify the conventional sequences ${ }^{44}$ by inserting a saturation procedure applied to carbon-13 prior to the mixing interval. This has two advantages: i) the state of carbon polarization is perfectly defined at the beginning of the mixing period (it is actually zero), ii) because time averaging is needed, the experiment can be repeated according to the proton relaxation time, usually much shorter than the carbon relaxation time. Furthermore, the $180^{\circ}$ phase alternation of the second proton pulse along with the alternation of the acquisition sign has the effect of cancelling any recovery of carbon polarization by carbon relaxation during the mixing time. 
As a consequence, pure nOe spectra are obtained.

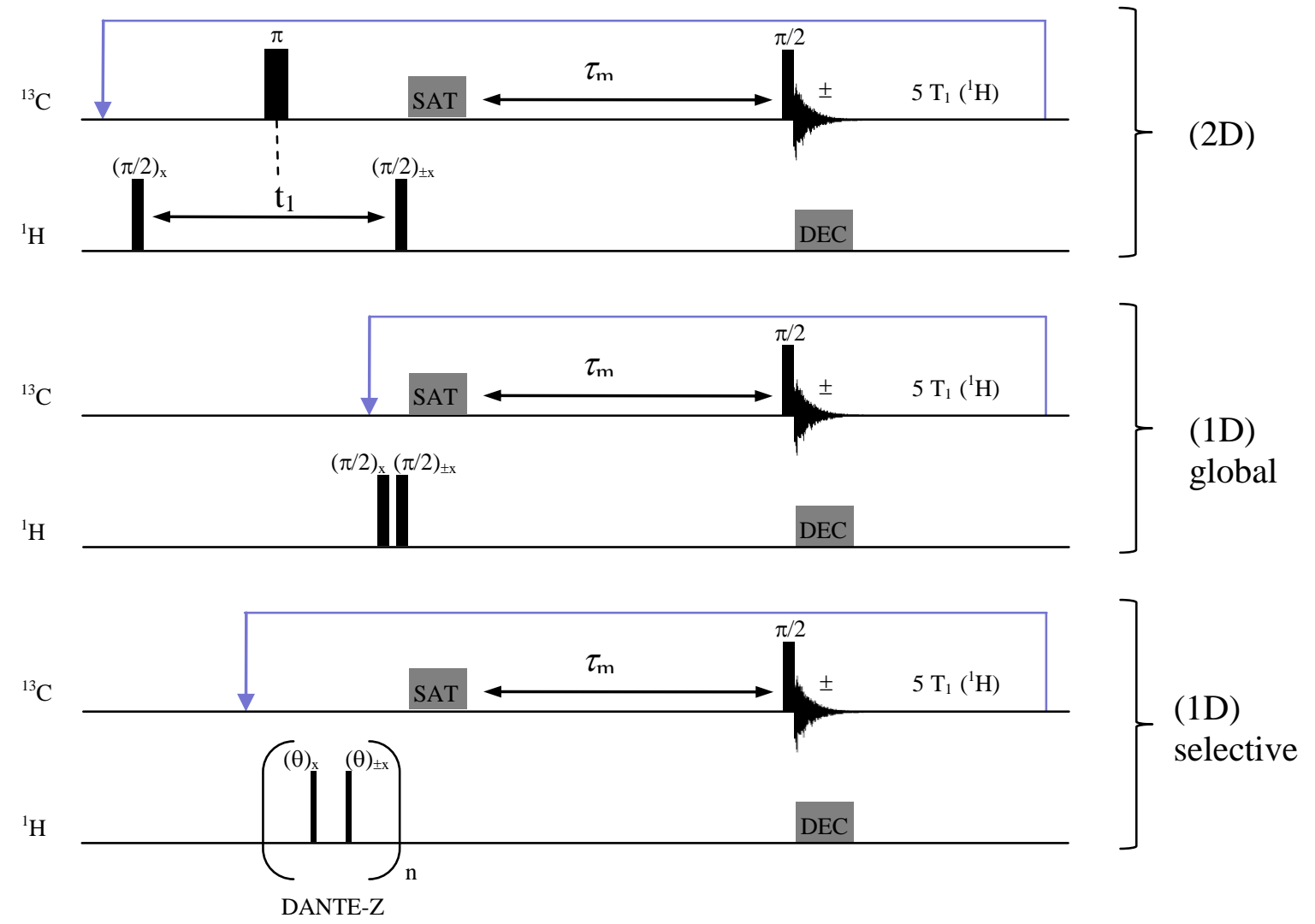

Figure 9. Three possible modes of the HOESY experiment.

In the $2 \mathrm{D}$ mode, the evolution time $\mathrm{t}_{1}$ plays its usual role for proton chemical shift labelling. The central $\pi$ pulse refocuses all proton-carbon $J$ couplings (decoupling pulse), so that the $v_{1}$ dimension corresponds to proton chemical shifts, while the $v_{2}$ dimension (physically detected) corresponds to carbon chemical shifts. Without that $\pi$ pulse, the correlation peaks appear at the position of the ${ }^{13} \mathrm{C}$ satellites in the proton spectrum, that is at more than $60 \mathrm{~Hz}$ on either side of the proton resonance. This can be a serious advantage for detecting remote correlations ${ }^{45,46}$ which appear precisely at the proton resonance position. Amplitude modulation occurs in $t_{1}$ so that a two-dimensional spectrum in the absorption mode can be obtained, facilitating the experimental construction of build-up curves (signal amplitude as a function of the mixing time; see figure 10). Thus an accurate measurement of the cross- 
relaxation rates can be achieved.

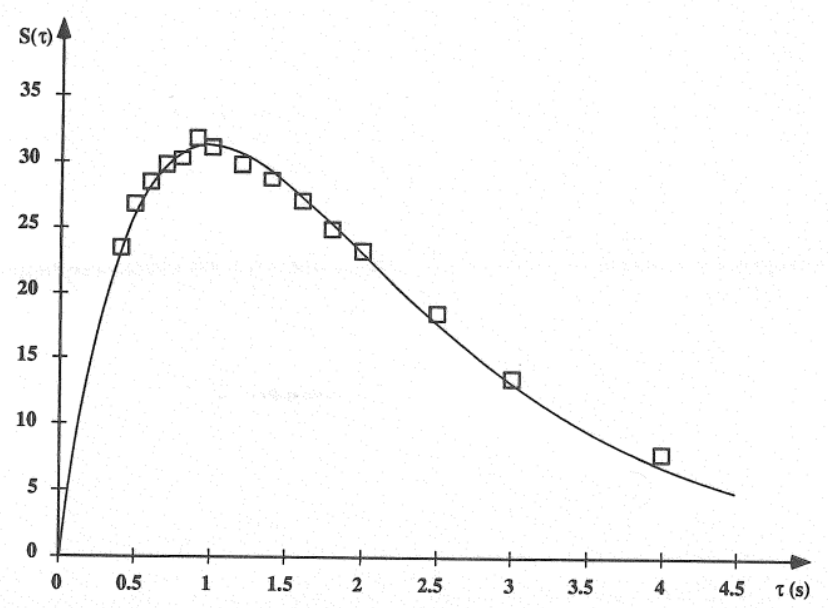

Figure 10. A typical HOESY build-up curve.

Whatever the method for extracting the cross-relaxation rates will be, a reference spectrum is needed. Actually it is furnished by the gated decoupling experiment shown at the bottom of figure 8 with the same number of scans than in the HOESY experiment. Let us denote by $I_{e q}^{C}$ the quantity which is measured that way. For a first estimation of the cross-relaxation rate, we can have recourse to the initial approximation which makes use of the data points corresponding to small values of the mixing time and seemingly varying linearly with the latter. Referring to equation (22), these data points can be exploited to first order as indicated below

$$
I_{z}^{C}\left(\tau_{m}\right)=I_{z}^{C}(0)+\left(\frac{d I_{z}^{C}}{d t}\right)_{t=0} \tau_{m}=4 \sigma_{e q}^{C} \tau_{m}
$$

For deriving the above equation, we have taken into account the phase cycling of figure (9) and the fact that $I_{e q}^{H}=4 I_{e q}^{C}$. The interesting result of (44) is that the cross-relaxation rate can be deduced from the initial slope of the plot of $I_{z}^{C} / I_{e q}^{C}$ as a function of $\tau_{m}$. This initial slope is equal here to $4 \sigma$ and would become $\sigma / 4$ if we would attempt to perform the experiment the other way, i.e. from carbon-13 toward proton. Anyway, when one has at hand the full buildup curve, it is recommended to fit the experimental data according to equations (22) or 
possibly to equations (27) if one suspects a contribution from csa-dipolar cross-correlation rates. In the former case, this will provide not only the cross-relaxation rate $\sigma$ (the parameter of interest) with an improved accuracy (because it is always difficult to decide what is the best initial slope) but also the longitudinal relaxation rates $R_{1}^{C}$ and $R_{1}^{H}$. Notice that $R_{1}^{H}$ can be affected by proton-proton cross-relaxation rates among the proton system ${ }^{46}$ (the so-called spin diffusion phenomenon). Anyhow, the problem of sensitivity, related to the observation of carbon-13 (the low gyromagnetic constant nucleus), may be addressed. Inverse twodimensional HOESY experiments ${ }^{47-50}$ have been proposed. They consist in transferring nOe from the low gyromagnetic constant nucleus to the more sensitive high gyromagnetic constant nucleus which is detected. In principle this should lead to a sensitivity increase by a factor of 16 in the case of the pair ${ }^{13} \mathrm{C}-{ }^{1} \mathrm{H}$. However, as the ${ }^{1} \mathrm{H}_{-}{ }^{13} \mathrm{C}$ cross-relaxation rate is 16 times smaller than the ${ }^{13} \mathrm{C}-{ }^{1} \mathrm{H}$ cross-relaxation rate (see above), there is no net sensitivity increase and these experiments are of little value as far as sensitivity is concerned. Moreover, the gradients used in such experiments for suppressing unwanted signals and selecting coherences of interest may entail a loss of sensitivity by a factor of two, which must be further multiplied by the increase of noise associated with detection at a higher frequency. However, in particular instances, inverse HOESY experiments can reveal correlations not visible in the direct HOESY experiments ${ }^{48,50}$. The true inverse HOESY experiment involves, by means of INEPT sequences, proton polarization transfer prior to the mixing time and proton detection together with the use of gradients as indicated above. This experiment has however been essentially devised for large biomolecules such as proteins ${ }^{51}$. As a matter of fact, because the INEPT transfer process is based on a well defined (large) $\mathrm{J}$ coupling, it is well suited for carbon-13 or nitrogen-15 directly bound to (a) proton(s) and leads the cross-relaxation rate associated with the $\mathrm{CH}$ or $\mathrm{NH}$ bond. The method has been widely used for the amide proton of proteins ${ }^{32}$ but, to the best of our knowledge, it has never been applied to the measurement 
of proton-carbon cross-relaxation rates in the case or small or medium size molecules. Nevertheless, it could probably be useful outside the extreme narrowing regime, along with measurements performed at different magnetic field values.

In difficult situations (weak cross-relaxation rates or strong overlap in the proton spectrum), when using carbon-13 detected HOESY experiments, one faces the unavoidable sensitivity problem. This problem can be alleviated by one-dimensional experiments, the easiest of which is shown in the middle of figure 9. It is a global experiment, in the sense that the proton chemical shift labelling has been suppressed. As a consequence, the measured quantity is the sum of the cross-relaxation rates arising from all protons prone to interact by dipolar coupling with a given carbon-13. It can be noticed that the basic phase cycling (essential for a proper interpretation of the experimental data) has been maintained in this sequence. This experiment can be useful as a prelude of a two-dimensional HOESY experiment requiring the accumulation of many transients, in order to make sure that the spectrometer occupation for a long time will not be wasted. It can also be used quantitatively for carbons experiencing a single dipolar interaction with protons. In that case, it would provide a piece of information comparable to that provided by the nOe factor. Another one-dimensional experiment is a selective one $\mathrm{e}^{40}$ as shown in the bottom of figure 9. This sequence allows one to determine the cross-relaxation rate produced by the selected proton. The DANTE-Z technique ${ }^{52}$ is particularly well suited for achieving the selectivity process because it involves inherently the basic phase cycling of the HOESY experiment. Of course, other selective schemes can be used, combined for instance with a TOCSY sequence which can be valuable when the proton of interest $(\mathrm{H})$ is not well separated from other multiplets. In that case, if $\mathrm{H}$ belongs to a $\mathrm{J}$ coupling network possessing an isolated proton $\left(\mathrm{H}^{\prime}\right), \mathrm{H}^{\prime}$ is selected at the outset and its polarization is transferred to $\mathrm{H}$ by the TOCSY sequence just prior to the mixing interval of the HOESY experiment ${ }^{53}$. 


\section{Examples}

\subsection{Stereo and conformational studies}

Early conformational studies by HOESY experiments are illustrated by the work of Batta and Köver $^{54}$ who were able to access oligosaccharide sequencing and conformational distribution around the glycosidic bond in model compounds. These determinations make use of relayed proton-proton-carbon cross-relaxation.

Another good example of what can be gained from HOESY experiments is provided by the work of Ancian et al. ${ }^{53}$ about the preferential conformation of uridine in water. Through the TOCSY-HOESY experiment above described, these authors were able to show unambiguously that the torsion angle of the uracil group around the glycosidic bond lies in a range corresponding to the anti-form of the pyrimidine base with respect to the furanose ring. The discrimination between $\mathrm{E}$ and $\mathrm{Z}$ isomers of the compound shown in figure 11 constitutes a further example of the interest of selective HOESY experiments ${ }^{55}$.

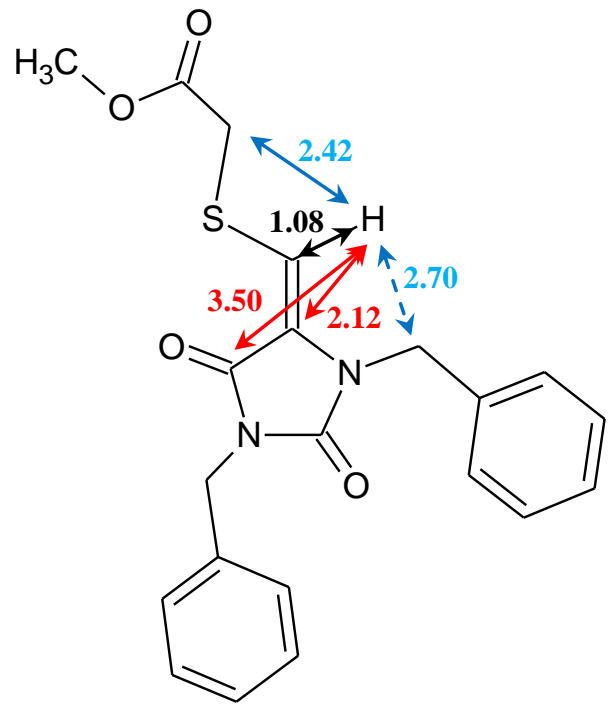

$\underline{E}$

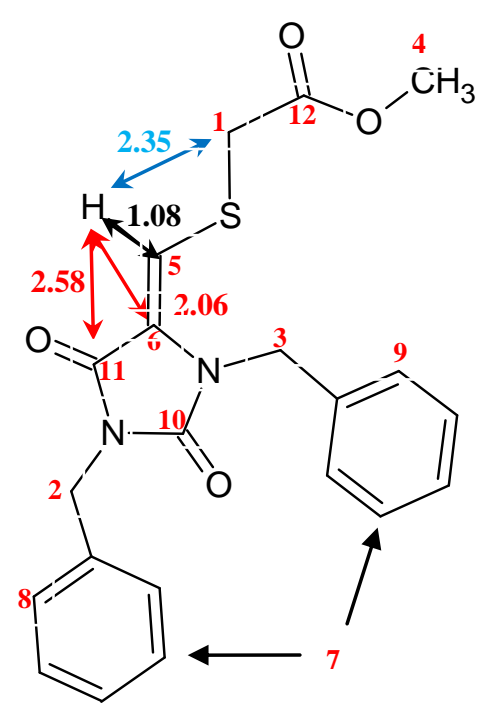

$\underline{Z}$

Figure 11. Two isomers with distances (in $\AA$ ) obtained by quantum chemistry calculations. Solid lines: proton-carbon distances. Dashed lines: proton-proton distances. 
This problem cannot be solved by the usual NMR experiments (COSY, NOESY, HSQC, HMBC...). It turns out that the selective HOESY experiment (figure 9, bottom) applied to proton $\mathrm{H}_{5}$ provides an unambiguous answer: for both isomers, $\mathrm{C}_{6}$ is close to $\mathrm{H}_{5}$, but for the $\mathrm{E}$ isomer $\mathrm{C}_{11}$ is remote from $\mathrm{H}_{5}$ whereas, in the $\mathrm{Z}$ isomer, $\mathrm{H}_{5}$ is relatively close to $\mathrm{C}_{11}$ yet less close than $\mathrm{C}_{6}$. It is exactly what is shown by figure 12 where the peak intensities are in the ratio of the distances (given in figure 11) to the power 6. It can therefore be concluded that we are in the presence of the $\mathrm{Z}$ isomer.

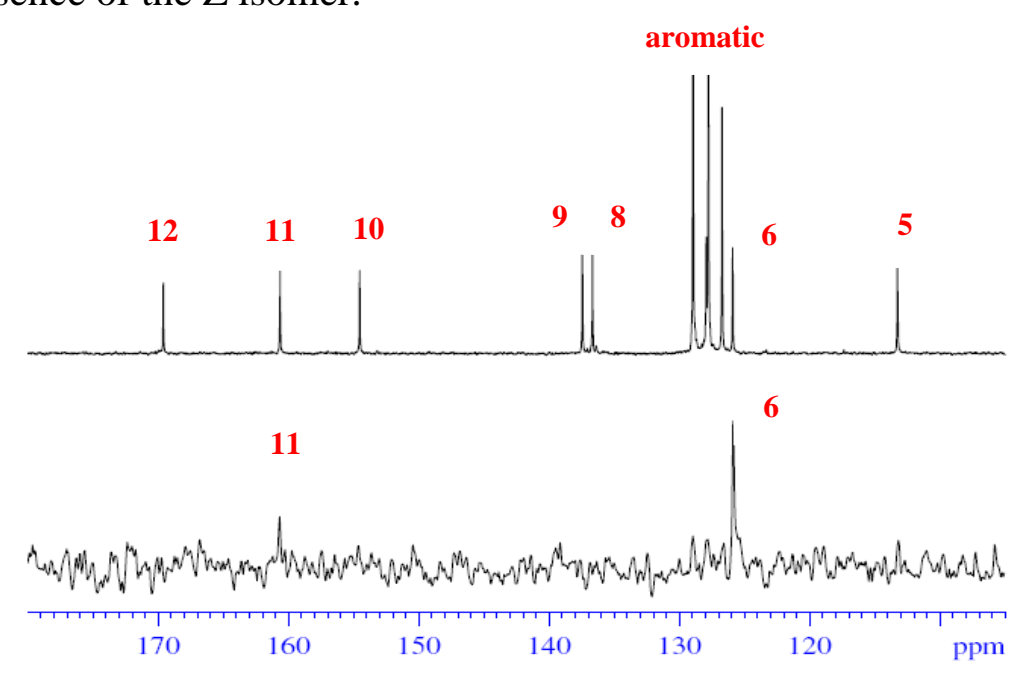

Figure 12. The ${ }^{13} \mathrm{C}$ responses (bottom) obtained by selectively inverting $\mathrm{H}_{5}$ (bound to a carbon-12); they lead to the cross-relaxation rates $\sigma_{H_{5}-C_{6}}$ and $\sigma_{H_{5}-C_{11}}$. A reference carbon-13 spectrum is shown at the top.

As a final example of this section, we present a HOESY two-dimensional experiment of aqueous micellized sodium octanoate ${ }^{46}$. In addition to one bond ${ }^{1} \mathrm{H}^{13}{ }^{13} \mathrm{C}$ correlation peaks, figure 13 exhibits remote correlation peaks which indicate some spatial proximity between a given carbon and protons bound to a nearby carbon. Build-up curves (not shown) have been obtained at two different values of the magnetic field since, as expected, a slow motion corresponding to the micelle overall motion is superposed to local fast motions (segmental and rotational isomerism). The cross-relaxation rates deduced from these build-up curves can be interpreted according to the Lipari-Szabo approach which involves the correlation time $\tau_{s}$ associated with the global micelle tumbling, the correlation time $\tau_{f}$ associated with the fast 
local motions and an order parameter $S$ which describes the restriction of the latter motions (see equation (39)).

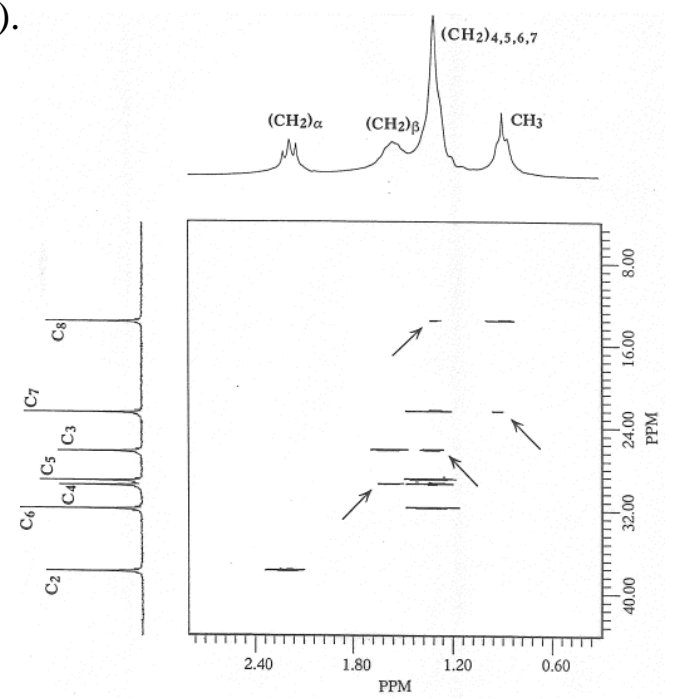

Figure 13. The HOESY two-dimensional spectrum of micellized sodium octanoate in aqueous solution. Besides one-bond carbon-proton correlations, remote correlations are observed (marked by an arrow).

Taking into account spin diffusion among the proton spin system (multiple proton-proton cross relaxation rates), one obtain order parameters in agreement with those derived from extensive carbon-13 longitudinal relaxation measurements (at different magnetic fields). More interesting are the remote correlations, two of them being shown in more detail in figure 14 . It can be seen that they are relatively intense and devoid of symmetry (compare $\mathrm{H}_{3}-\mathrm{C}_{4}$ and $\mathrm{H}_{4}$ $\left.\mathrm{C}_{3}\right)$.

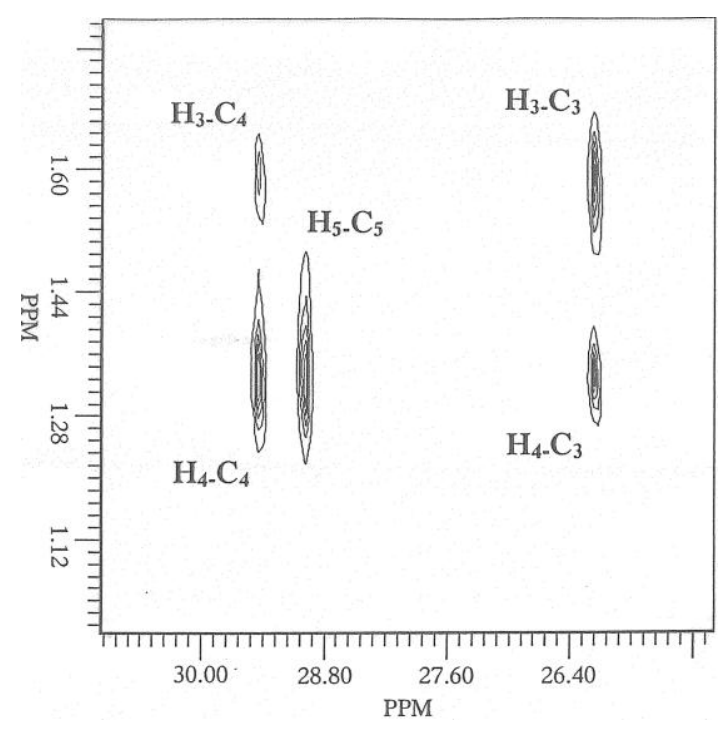

Figure 14. An excerpt of figure 13 showing peaks associated with remote correlations. 
Attempts to interpret the corresponding build-up curves according to the Lipari-Szabo approach lead to inconsistent results (for instance, order parameters greater than unity). This indicates that these remote correlations are probably not of intramolecular origin but would rather arise from intermolecular dipolar interactions which could become significant when some contacts exist between neighboring aliphatic chains. This can only occur for parts of the chain presenting some flexibility (due to rotational isomerism or segmental motions). This flexibility may not be the same at the $\mathrm{C}_{3}$ or $\mathrm{C}_{4}$ levels and this would explain the different intensities of the $\mathrm{H}_{3}-\mathrm{C}_{4}$ and $\mathrm{H}_{4}-\mathrm{C}_{3}$ peaks. Thus, in that case, the HOESY experiment brings information about aliphatic chain mobility. Indeed, the part of the chain located near the polar head is known to be rigid and this is confirmed by the absence of $\mathrm{H}_{2}-\mathrm{C}_{3}$ and $\mathrm{H}_{3}-\mathrm{C}_{2}$ correlations.

\subsection{Geometrical determinations}

During the seventies and the eighties, $\mathrm{C}-\mathrm{H}$ distances in various medium size molecules were determined through proton-carbon-13 nOe cross-relaxation rates in a semi-quantitative way. These determinations followed the release of the seminal book by Noggle and Schirmer ${ }^{56}$ and, most of the time, rested on the assumption of a single correlation time describing the overall motion of the molecule under investigation. These studies relied on global and selective nOe's. A complete bibliography can be found in the paper by Batta et al. ${ }^{57}$, who, in addition, treated an AMX spin system where A and M stand for two J-coupled protons and X for the observed carbon-13.

The previous approach is valid as long as the molecular reorientation can be described by a single correlation time. This excludes molecules involving internal motions and/or molecular shapes which cannot, to a first approximation, be assimilated to a sphere. Due to its shape, the molecule shown in figure 15 cannot evidently fulfil the latter approximation and is illustrative 
of the potentiality of HOESY experiments as far as carbon-proton distances and the anisotropy of molecular reorientation are concerned $\mathrm{d}^{45,58}$.

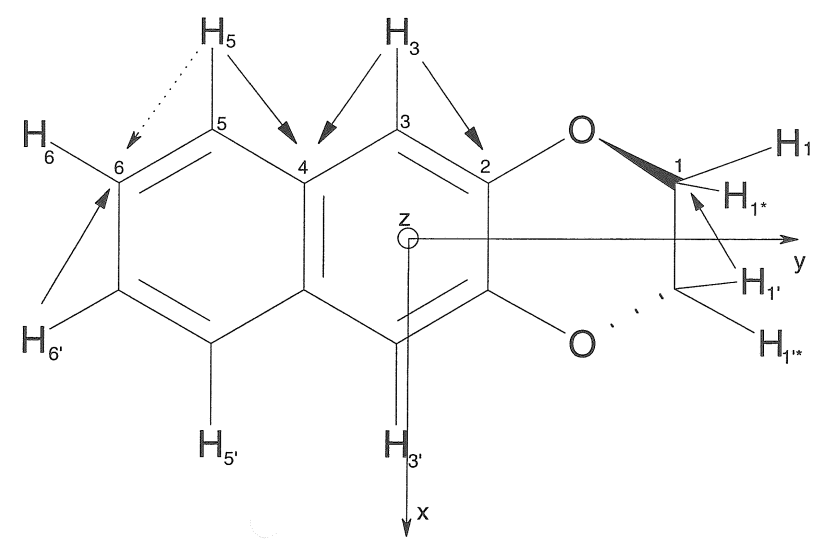

Figure 15. The model molecule used to demonstrate the possibilities of HOESY experiments in terms of carbon-proton distances and reorientational anisotropy. To a first approximation, the molecule is devoid of internal motions and its symmetry determines the principal axis of the rotation-diffusion tensor. Note that $\mathrm{H}_{1}, \mathrm{H}_{1} * \mathrm{H}_{1}, \mathrm{H}_{1^{\prime *}}$ are non equivalent. The arrows indicate remote correlations.

The HOESY spectrum is displayed in figure 16. It has been obtained in the "J-separated" mode, $i$.e. without the central $\pi$ pulse in the $t_{1}$ interval (see figure 9 , top) in such a way that direct correlations (one bond) appear at the location of carbon-13 satellites whereas possible remote correlations are visible at the proton resonance frequency (see section 5.3). A first estimate of the molecular geometry can be deduced from quantum chemical calculations. Direct correlations (which involve one bond C-H distances around $1.1 \AA$ ) can then be employed for deriving the three rotational diffusion coefficients which reveal a strong reorientational anisotropy: expressed in terms of correlation times, this leads to $\tau_{\mathrm{x}}=18 \pm 5 \mathrm{ps}$, $\tau_{\mathrm{y}}=2.6 \pm 0.2 \mathrm{ps}$ and $\tau_{\mathrm{z}}=10.8 \pm 1.8 \mathrm{ps}$. Evidently, it is out of the question to use the approximation of a single correlation time and equations (34)-(36) must be used. The angles appearing in these equations can be assumed from the quantum mechanical calculations and the crossrelaxation rates derived from the build-up curves pertaining to remote correlations allow one to derive the relevant distances. They are collected in table 1 and compared to those given by quantum mechanical calculations and crystallography. 


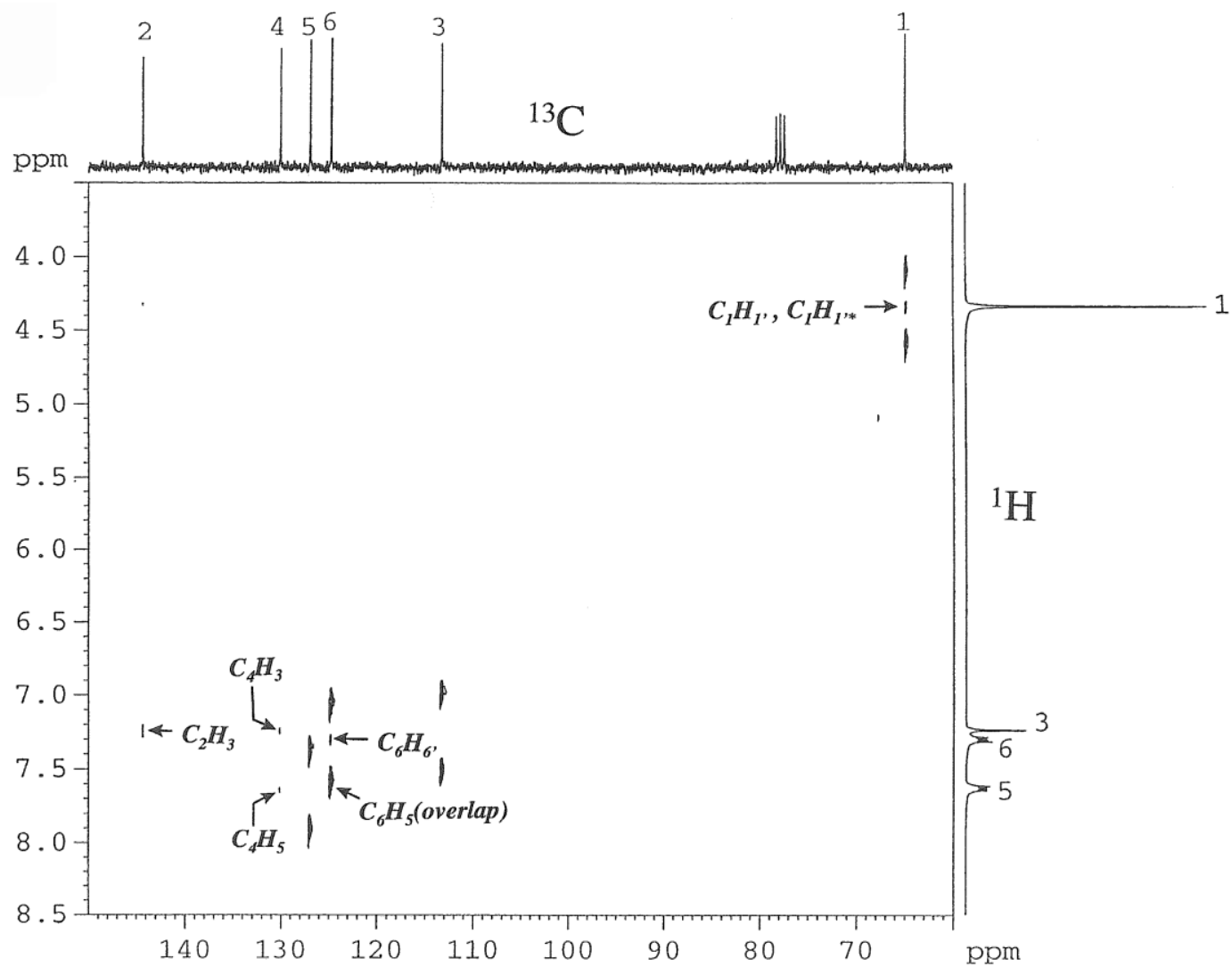

Figure 16. The J-separated HOESY spectrum of the molecule shown in figure 15. Direct (onebond) correlations are located at the position of ${ }^{13} \mathrm{C}$ satellites in the proton spectrum. Arrows indicate remote correlations.

Table 1. Distances in $\AA$ derived from the HOESY experiment ( $r_{\mathrm{NMR}}$ and compared to results from crystallography $\left(r_{\mathrm{RX}}\right)$ and quantum mechanical calculations $\left(r_{\mathrm{QM}}\right)$.

\begin{tabular}{|c|c|c|c|}
\hline vector & $r_{\mathrm{NMR}}$ & $r_{\mathrm{RX}}$ & $r_{\mathrm{QM}}$ \\
\hline $\mathrm{C}_{1} \mathrm{H}_{1^{\prime}}$ & 2.02 & 2.07 & 2.20 \\
\hline $\mathrm{C}_{1} \mathrm{H}_{1^{\prime *}}$ & 1.96 & & 2.21 \\
\hline $\mathrm{C}_{2} \mathrm{H}_{3}$ & 2.32 & 2.24 & 2.16 \\
\hline $\mathrm{C}_{4} \mathrm{H}_{3}$ & 2.14 & 2.07 & 2.19 \\
\hline $\mathrm{C}_{4} \mathrm{H}_{5}$ & 1.81 & 2.04 & 2.19 \\
\hline $\mathrm{C}_{6} \mathrm{H}_{6^{\prime}}$ & 2.19 & 2.24 & 2.18 \\
\hline
\end{tabular}

The agreement between the three techniques is rather good, bearing in mind that quantum mechanical calculations totally ignore molecular vibrations and that different vibrational averaging should be performed for $r_{\mathrm{NMR}}$ and $r_{\mathrm{RX}}$.

In the case of a molecule with low symmetry and a reasonably known geometry, the inverse approach can be considered, i.e. determine not only $\tau_{\mathrm{x}}, \tau_{\mathrm{y}}$ and $\tau_{\mathrm{z}}$, but also the principal axis system of the rotation-diffusion tensor. The molecule shown in figure 17 is a good example of 
such a study ${ }^{59}$ : direct and remote correlations of a HOESY spectrum, along with an assumed geometry, lead to the orientation of the rotation-diffusion tensor principal axis system. Interestingly, it can be seen that this orientation has nothing to do neither with the inertia tensor, nor with the dipole moment direction. Again the reorientation is here strongly anisotropic: $\tau_{\mathrm{x}}=12.0 \pm 2.5 \mathrm{ps}, \tau_{\mathrm{y}}=32 \pm 04 \mathrm{ps}$ and $\tau_{\mathrm{z}}=4.1 \pm 1.0 \mathrm{ps}$

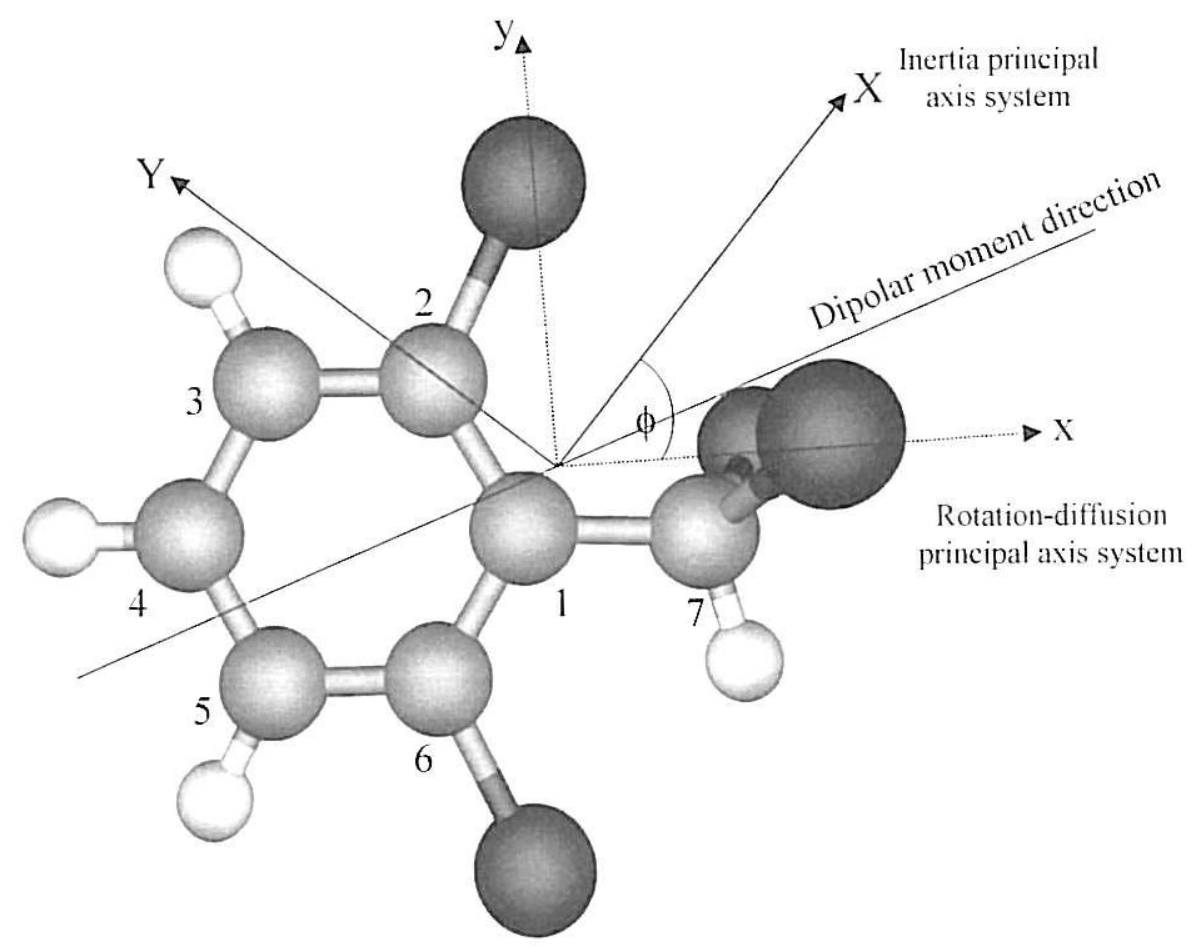

Figure 17. The $\alpha, \alpha, 2,6$ tetrachlorotoluene. Small letters refer to the rotation-diffusion principal axis system.

Still, in view of determining accurate $\mathrm{C}-\mathrm{H}$ distances, a promising approach has been developed by Kowalewski and coworkers ${ }^{60}$. It makes use of what these authors call a "cryosolvent", a mixture of $\mathrm{D}_{2} \mathrm{O}$ and dimethyl sulfoxide (in a 2:1 molar ratio) which has the property of being very viscous at low temperature. As a consequence, any molecule in this cryosolvent is outside the extreme narrowing regime and relaxation parameters become frequency dependent. Their dependence upon the magnetic field will yield the correlation time(s) whereas their amplitudes lead to the interatomic distances. As an unambiguous example, these authors choose the hexamethylenetetramine (figure 18) which possesses a high 
symmetry so that a single correlation time is required. Moreover, for carbon-13, the predominant relaxation mechanism should be the dipolar interaction with protons. With the longitudinal relaxation time and the nOe factor measured at two different magnetic fields, a C-H distance of $1.142 \AA$ was found. This distance reduces to $1.108 \AA$ after vibrational averaging and compares very well with other experimental determinations. This study validates the capability of spin relaxation for accessing molecular geometry. This feature has been confirmed and complemented by the measurement of cross-correlation relaxation rates ${ }^{61}$.

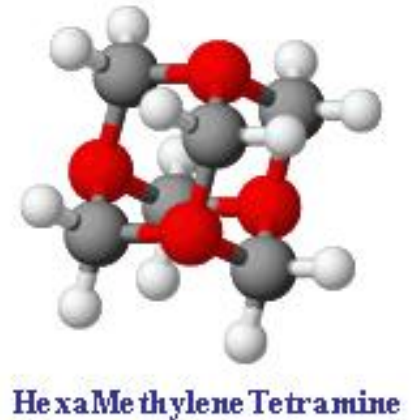

Figure 18. The molecule studied in a cryosolvent in order to determine by carbon-13 relaxation an accurate $\mathrm{C}-\mathrm{H}$ distance.

\section{Conclusion}

Through this review, it can be seen that the proton-carbon nOe have not been used as it probably deserves, as far as small or medium size molecules are concerned. As a possible perspective, one could envision extensive HOESY measurements at very high field (even combined with proton detection, a possibility for improving sensitvity not yet explored in the case of small or medium size molecules) in order to improve the spectral resolution and to obtain more and more remote correlations which could be useful for refining the geometrical parameters. Still toward this objective, another valuable approach is probably the use of cyosolvents. Altogether, one could dream of a sort of spin relaxation NMR crystallography. 
This work is part of the ANR project MULOWA (Grant Blan08-1_325450)

\section{REFERENCES}

1. F. Bloch, Phys. Rev., 1946, 70, 460.

2. D. Neuhaus and M.P. Williamson, The nuclear Overhauser effect in structural and conformational analysis. Verlag Chemie, New york, 1989.

3. J. Jeener, B.H. Meier, P. Bachmann and R.R. Ernst, J. Chem. Phys., 1979, 71, 4546.

4. G.C. Levy, R.L. Lichter, G.L. Nelson, Carbon-13 nuclear magnetic resonance spectroscopy (second edition). Wiley, New york, 1980.

5. D. Canet, Nuclear Magnetic Resonance: Concepts and Methods. Wiley, Chichester, 1996.

6. J. Kowalewski and L. Mäler, Nuclear spin relaxation in liquids: theory, experiments and applications. Taylor \& Francis, New York, 2006.

7. I. Solomon, Phys. Rev., 1955, 99, 559D.E. Woessner, J. Chem. Phys., 1962, 37, 647.

8. A.W. Overhauser, Phys. Rev., 1953, 92, 401.

9. O.W. Sorensen, G.W. Eich, M.H. Levitt, G. Bodenhausen and R.R. Ernst, Prog. NMR Spectr., 1983, 16, 163

10. A. Kumar, R.C.R. Grace and P.K. Mahdu, Prog. NMR Spectr., 2000, 37, 191.

11. L.G. Werbelow and D.M. Grant, Adv. Magn. Reson., 1977, 9, 189.

12. D. Canet, Prog. NMR Spectr., 1989, 21, 203.

13. L. Nordstierna, P.V. Yushmanov and I. Furó, J. Chem. Phys., 2006, 125, 074704.

14. F. Perrin, J. Phys. Rad., 1934, V, 33.

15. D.E. Woessner, J. Chem. Phys., 1962, 37, 647.

16. W.T. Huntress, Jr., J. Chem. Phys., 1968, 48, 3524.

17. P. S. Hubbard, J. Chem. Phys., 1970, 52, 563.

18. D. Canet, Conc. Magn. Reson., 1998, 10, 291.

19. A. Ericsson, J. Kowalewski, T. Liljefors and P. Stilbs, J. Magn. Reson., 1980, 38, 9.

20. D.E. Woessner, in: Encyclopedia of Nuclear Magnetic Resonance, D.M. Grant and R.K. Harris (eds.), Wiley, Chichester, 1996, p.1068.

21. V.A. Daragan and K.H. Mayo, Prog. NMR Spectr., 1997, 31, 63.

22. M.W.F. Fischer, A. Majumdar and E.R.P. Zuiderweg, Prog. NMR Spectr., 1998, 33, 207.

23. D.M. Korzhnev, M. Billeter, A.S. Arseniev and V.Y. Orekhov, Prog. NMR Spectr., 2001, 38, 197.

24. P. Luginbühl and K. Wüthrich, Prog. NMR Spectr., 2002, 40, 199.

25. G. Lipari ans A. Szabo, J. Am. Chem. Soc., 1982, 104, 4546.

26. H. Wennerström, B. Lindman, O. Söderman, T. Drakenberg and J.B. Rosenholm, J. Am. Chem. Soc., 1979, 101, 6860.

27. D. Canet, S. Bouguet-Bonnet and P. Mutzenhardt, Conc. Magn. Reson., 2003, 19A, 65.

28. D. Frueh, Prog. NMR Spectr., 2002, 41, 305.

29. R.L. Vold, J.S. Waugh, M.P. Klein and D.E. Phelps, J. Chem. Phys., 1968, 48, 3831.

30. D. Canet, G.C. Levy and I.R. Peat, J. Magn. Reson., 1975, 18, 199.

31. R. Freeman and H.D.W. Hill, J. Chem. Phys., 1971, 54, 3367.

32. F. Ferrage, A. Reichel, S. Battacharya, D. Cowburn and R. Ghose, J. Magn. Reson., 
2010, 207, 294.

33. R. Freeman, H.D.W. Hill and R. Kaptein, J. Magn. Reson., 1972, 7, 327.

34. D. Canet, J. Magn. Reson., 1976, 23, 361.

35. P.L. Rinaldi, J. Am. Chem. Soc., 1983, 105, 5167.

36. C. Yu and G.C. Levy, J. Am. Chem. Soc., 1983, 105, 6994.

37. C. Yu and G.C. Levy, J. Am. Chem. Soc., 1984, 106, 6533.

38. W. Bauer and P. R. von Schleyer, Magn. Reson. Chem., 1988, 26, 827.

39. H. Seba and B. Ancian, J. Chem. Soc., Chem. Comm., 1990, 996.

40. D. Canet, N. Mahieu and P. Tekely, J. Am. Chem. Soc., 1992, 114, 6190.

41. A. Belmajdoub, N. Mahieu, P. Tekely and D. Canet, J. Phys. Chem., 1992, 96, 1011.

42. N. Mahieu, P. Tekely and D. Canet, J. Phys. Chem., 1993, 97, 2764.

43. I. Furó, P. Mutzenhardt and D. Canet, J. Am. Chem. Soc., 1995, 117, 10405.

44. K.B. Köver and G. Batta, Prog. NMR Spectr., 1987, 19, 223.

45. P. Mutzenhardt, O. Walker, D. Canet, E. Haloui and I. Furó, Molec. Phys., 1998, 94, 565.

46. P. Palmas, P. Tekely, P. Mutzenhardt and D. Canet, J. Chem. Phys., 1993, 99, 4775.

47. W. Bauer, Magn. Reson. Chem., 1996, 34, 532.

48. K. Stott and J. Keeler, Magn. Reson. Chem., 2003, 41, 554.

49. T.M. Alam, D.M. Pedrotty and T.J. Boyle, Magn. Reson. Chem., 2002, 40, 361.

50. O. Walker, P. Mutzenhardt and D. Canet, Magn. Reson. Chem., 2003, 41, 776.

51. L.E. Kay, D.A. Torchia and A. Bax, Biochemistry, 1989, 28, 8972.

52. D. Boudot, D. Canet, J. Brondeau and J.C. Boubel, J. Magn. Reson., 1989, 83, 428.

53. B. Ancian, D. Canet and P. Mutzenhardt, Chem. Phys. Lett., 2001, 336, 410.

54. Gy. Batta and K.E. Köver, Magn. Reson. Chem., 1988, 26, 852.

55. M. Yemloul, S. Bouguet-Bonnet, L.A.C. Ba, G. Kirsch and D. Canet, Magn. Reson. Chem., 2008, 46, 939.

56. J.H. Noggle and R.E. Schirmer, The Nuclear Overhauser Effect. Academic Press, New York, 1971.

57. G. Batta, K.E. Köver and Z. Madi, J. Magn. Reson., 1987, 73, 477.

58. O. Walker, P. Mutzenhardt, J.P. Joly and D. Canet, Chem. Phys. Lett., 2002, 357, 103.

59. O. Walker, P. Mutzenhardt, E. Haloui, J.-C. Boubel and D. Canet, Molec. Phys., 2002, 100, 2755.

60. J. Kowalewski, M. Effemey and J. Jokisaari, J. Magn. Reson., 2002, 157, 171.

61. L. Ghalebani, P. Bernatowicz, S. N. Aski and J. Kowalewski, Conc. Magn. Reson., 2006, 30A, 100. 